\title{
THE HUA SYSTEM ON IRREDUCIBLE HERMITIAN SYMMETRIC SPACES OF NONTUBE TYPE
}

\author{
DARIUSZ BURACZEWSKI
}

\begin{abstract}
Let $G / K$ be an irreducible Hermitian symmetric space of noncompact type. We study a $G$-invariant system of differential operators on $G / K$ called the Hua system. It was proved by K. Johnson and A. Korányi that if $G / K$ is a Hermitian symmetric space of tube type, then the space of Poisson-Szegö integrals is precisely the space of zeros of the Hua system. N. Berline and M. Vergne raised the question about the nature of the common zeros of the Hua system for Hermitian symmetric spaces of nontube type. In this paper we show that these are exactly the pluriharmonic functions.
\end{abstract}

\section{INTRODUCTION}

Let $G / K$ be an irreducible Hermitian symmetric space of noncompact type and let $\left\{E_{j}\right\}$ be an orthonormal basis of $\mathfrak{p}^{+}$. The Hua system, as defined in [JK], is

$$
\mathcal{H}(F)=\sum_{j, k} E_{j} E_{k}^{*} F \otimes\left[E_{j}, E_{k}^{*}\right] .
$$

After a number of partial results, the earliest going back to Hua, the fundamental theorem concerning the Hua system, proved by K.Johnson and A. Korányi is:

Theorem 1.2. (K. Johnson, A. Korányi, 1980) A function F on a Hermitian symmetric space of tube type satisfies $\mathcal{H}(F)=0$ if and only if it is the Poisson-Szegö integral of a hyperfunction on the Shilov boundary of $G / K$.

Soon after the result of K. Johnson and A. Korányi appeared, N. Berline and M. Vergne $[\mathrm{BV}]$ proved that (1.1) does not annihilate the Poisson-Szegö kernel on type two Hermitian symmetric spaces and described a third order system that characterizes Poisson-Szegö integrals for those spaces, and they raised the question about the nature of the common zeros of the Hua system for the type two Hermitian symmetric spaces, a question that has remained opened for twenty years.

The aim of this paper is to prove that the zeros of the Hua system on type two Hermitian symmetric spaces are the pluriharmonic functions (see section 4):

Theorem 1.3. Let $F$ be a real valued function on a non-tube irreducible Hermitian symmetric space. Then $\mathcal{H}(F)=0$ if and only if $F$ is pluriharmonic.

The author was partly supported by KBN grant 5P03A02821, Foundation for Polish Sciences, Subsidy 3/99, and by the European Commission via the TMR Network "Harmonic Analysis", contract no. ERB FMRX-CT97-0159. 
The system (1.1) annihilates holomorphic and antiholomorphic functions. The origin of (1.1) goes back to L. H. Hua [Hua], who in 1958 wrote a system that annihilates the Poisson-Szegö kernel on some classical domains. His formula was not exactly the one above, but for classical tube domains the zeros of both systems are the same. Then A. Korányi, E. Stein and J. Wolf obtained the formula for general tube domains and in an unpublished paper showed that the Poisson-Szegö kernel is harmonic with respect to the system (see e.g. $[\mathrm{JK}]$ ). The first results showing that differential equations actually characterize the class of Poisson-Szegö integrals were obtained in special cases [KM], [J1], [J2]. Finally in 1980 K. Johnson and A. Korányi proved Theorem (1.2).

For a particular case of functions having $L^{2}$ boundary values, the above theorem was proved in [BBDHPT]. The methods of [BBDHPT] make the utmost use of the strong growth restrictions and are not applicable here.

To treat zeros of $\mathcal{H}$ in full generality we combine two essential ingredients: the method of M.Lassalle $[\mathrm{L}]$ and the approach to pluriharmonic functions on symmetric Siegel domains developed in [DHMP], [BDH]. While the first one is based on the semi-simple group $G$, for the second one the use of the solvable Lie group $S$ acting simply transitively on the corresponding Siegel domain seems indispensable. The reason is that the $G$-invariant operators don't see pluriharmonicity while the $S$ invariant do ${ }^{1}$ The interplay between $S$ picture and $G$-picture is crucial for our story: the analysis is done on the group $S$ and the special structure of $S$ being the Iwasawa group is essential. That is why we describe so thoroughly both pictures: $G / K$ and $S$ and we pass from one to the other (sections 2-3).

M.Lassalle [L] reproved theorem (1.2) introducing new methods and, at the same time, cutting down the number of equations. We adopt his method to reduce the problem to bounded functions. Namely, we prove that a Hua harmonic function is $G$-harmonic (section 5$)^{2}$. To do so we use only a part of the system, the "strongly diagonal operators" (see section 4). These $r$ equations ( $r$ being the rank) correspond to the system $\mathcal{H}_{h}$ of M. Lassalle. Next we use Harish-Chandra theorem $[\mathrm{HC}]$ in order to expand $f$ in terms of its projections on spaces of $K$-finite functions $f_{\delta}$. Each of these functions is Hua harmonic, hence $G$-harmonic. A $K$-finite and $G$-harmonic function can be written as a Poisson integral of a continuous bounded function defined on the maximal Furstenberg boundary, therefore all functions $f_{\delta}$ are bounded.

After restricting to bounded functions we transfer our problem to Siegel domains. For this we pass to realization of $G / K$ as a Siegel domain ${ }^{c} \mathcal{D}$ in $\mathbb{C}^{m}$, which is described in section (3.3) following the Korányi-Wolf theory [KW]. However, for our purposes, we have to transform it further on in order to write strongly diagonal operators on the solvable Lie group $S$, the one that acts simply transitively on ${ }^{c} \mathcal{D}$ (section 6 ). This gives an extra advantage: the whole system may be replaced by strongly diagonal operators. In fact, we prove (see section 4):

\footnotetext{
${ }^{1}$ Bounded pluriharmonic functions are Poisson integrals i.e they are annihilated by all the $G$-invariant operators, but there are many $G$-harmonic functions that are not pluriharmonic.

${ }^{2}$ The fact that for type two domains Hua harmonicity implies $G$-harmonicity was mentioned without proof in $[\mathrm{JK}]$.
} 
Theorem 1.4. Let $F$ be a bounded real valued function on a non-tube Hermitian irreducible symmetric space. If $F$ is annihilated by the Laplace-Beltrami operator and the strongly diagonal Hua operators then $F$ is pluriharmonic.

Hence only $r+1$ operators are needed which is considerably less than in either JohnsonKorányi's or Lassalle's proof.

The rest of the proof uses $S$ (section 6). First we show that a bounded function annihilated by the strongly diagonal Hua operators is the Poisson-Szegö integral (see [BBDHPT], $[\mathrm{DHP}])$. Then, we notice that the Laplace-Beltrami operator $\Delta_{T}$ for the corresponding tube domain $T_{\Omega}$ is a linear combination of the above $r+1$ operators. Combining these two facts with Theorem (1.2) applied to $T_{\Omega}$ we obtain some more equations (see section $6)$.

Now the strategy is to single out operators whose common zeros have to be pluriharmonic. These operators, however, cannot be obtained directly as linear combinations of the ones studied so far. The method we apply is the induction on the rank of the domain. The crucial observation is that $S$ is a semi-direct product

$$
S=S^{r-1} S_{r}
$$

where $S^{r-1}$ is the group acting simply transitively on the Siegel domain of rank $r-1$ and $S_{r}$ the group acting simply transitively on the Siegel half plane $\mathcal{D}_{r}$ (biholomorphic to the complex ball). Since a part of the equations that we have at our disposal are on $S_{r}$, we restrict the function $F$ to $S_{r}$ and apply some Fourier analysis methods on the Heisenberg group (section 7). The induction produces equations to which we can apply the results of $[\mathrm{BDH}]$ and conclude that $F$ is pluriharmonic. Since this method requires that we deal with bounded functions not $L^{2}$, the analytic part here is somewhat more delicate than in [BBDHPT].

The author would like to express his deep gratitude to Ewa Damek for her numerous ideas, suggestions and corrections incorporated in the paper. Indeed, some parts of this paper are in fact a joint work. Also the author wishes to thank Aline Bonami, Jacques Faraut and Andrzej Hulanicki for their valuable comments.

\section{Preliminaries on Hermitian Symmetric SPACES}

Let $G / K$ be an irreducible Hermitian symmetric space of noncompact type and $G$ the connected component of its isometry group. $G$ is a centerless semisimple Lie group, and $K$ is its maximal compact subgroup. We need some standard notation concerning semisimple Lie groups and algebras. For more details we refer to $[\mathrm{H} 1],[\mathrm{Kn}]$ or $[\mathrm{K}]$.

2.1. Root space decomposition. Let $\mathfrak{g}$ and $\mathfrak{k}$ be the Lie algebras of respectively $G$ and $K$, and let $\theta: \mathfrak{g} \mapsto \mathfrak{g}$ be the Cartan involution on $\mathfrak{g}$ which is identity on $\mathfrak{k}$. If $\mathfrak{p}$ is the -1 eigenspace of $\theta$, we get the Cartan decomposition $\mathfrak{g}=\mathfrak{k} \oplus \mathfrak{p}$, where $[\mathfrak{k}, \mathfrak{p}]=\mathfrak{p}$ and $[\mathfrak{p}, \mathfrak{p}]=\mathfrak{k}$. Let $\mathfrak{g}^{\mathbb{C}}\left(\mathfrak{k}^{\mathbb{C}}, \mathfrak{p}^{\mathbb{C}}\right.$ resp.) denote the complexification of $\mathfrak{g}$ (k, $\mathfrak{p}$ resp.). We extend $\theta$ to be a complex linear involution on $\mathfrak{g}^{\mathbb{C}} . G^{\mathbb{C}}$ is the adjoint group of $\mathfrak{g}^{\mathbb{C}}$ with $K^{\mathbb{C}}$ the analytic subgroup corresponding to $\mathfrak{k}^{\mathbb{C}}$. 
$\mathfrak{u}=\mathfrak{k} \oplus i \mathfrak{p}$ is a compact real form of $\mathfrak{g}^{\mathbb{C}}$. Denote by $\tau$ the conjugation operator on $\mathfrak{g}^{\mathbb{C}}$ with respect to $\mathfrak{u}$. If $B$ is the Killing form on $\mathfrak{g}^{\mathbb{C}}$, then the bilinear form defined by $B_{\tau}(X, Y)=-B(X, \tau Y)$ is positive definite. $\sigma=\tau \theta=\theta \tau$ is the conjugation of $\mathfrak{g}^{\mathbb{C}}$ with respect to $\mathfrak{g}$. (Usually we shall write $\bar{E}$ instead of $\sigma E$.)

Choose a Cartan subalgebra $\mathfrak{h}$ in $\mathfrak{k}$. Then $\mathfrak{h}^{\mathbb{C}}$ is a Cartan subalgebra of $\mathfrak{g}^{\mathbb{C}}$. Define $\Delta$ to be the system of roots of $\mathfrak{g}^{\mathbb{C}}$ with respect to $\mathfrak{h}^{\mathbb{C}}$. Any root space $\mathfrak{g}^{\alpha}$ is contained either in $\mathfrak{k}^{\mathbb{C}}$ or in $\mathfrak{p}^{\mathbb{C}}$. In the first case $\alpha$ is called compact $(\alpha \in C)$ and in the second case noncompact $(\alpha \in Q)$. Clearly $\Delta$ is a disjoint union of $C$ and $Q$. For a fixed $\alpha \in \Delta$ there are $\widetilde{H}_{\alpha} \in i \mathfrak{h}, \widetilde{E}_{\alpha} \in \mathfrak{g}^{\alpha}, \widetilde{E}_{-\alpha} \in \mathfrak{g}^{-\alpha}$ such that

$$
\begin{aligned}
\alpha(H) & =B\left(H, \widetilde{H}_{\alpha}\right), \quad \text { for every } H \in \mathfrak{h}^{\mathbb{C}}, \\
{\left[\widetilde{E}_{\alpha}, \widetilde{E}_{-\alpha}\right] } & =\widetilde{H}_{\alpha}, \\
\tau \widetilde{E}_{\alpha} & =-\widetilde{E}_{-\alpha}, \\
B\left(\widetilde{E}_{\alpha}, \widetilde{E}_{-\alpha}\right) & =1,
\end{aligned}
$$

([H1], page 220). In particular, $\widetilde{H}_{\alpha}, \widetilde{E}_{\alpha}$ and $\widetilde{E}_{-\alpha}$ span a subalgebra of $\mathfrak{g}^{\mathbb{C}}$, isomorphic to $\mathfrak{s l}(2, \mathbb{C})$.

We define a Hermitian product on $\left(\mathfrak{h}^{\mathbb{C}}\right)^{*}$ by

$$
\langle\alpha, \beta\rangle=B\left(\widetilde{H}_{\alpha}, \widetilde{H}_{\beta}\right)=\alpha\left(\widetilde{H}_{\beta}\right)=\beta\left(\widetilde{H}_{\alpha}\right)
$$

for $\alpha, \beta \in\left(\mathfrak{h}^{\mathbb{C}}\right)^{*}$.

Let

$$
c_{\alpha}=\sqrt{\frac{2}{\alpha\left(\widetilde{H}_{\alpha}\right)}}=\sqrt{\frac{2}{\langle\alpha, \alpha\rangle}} .
$$

$c_{\alpha}$ is well defined because $\alpha\left(\widetilde{H}_{\alpha}\right)=B\left(\widetilde{H}_{\alpha}, \widetilde{H}_{\alpha}\right)>0$. Clearly $c_{\alpha}=c_{-\alpha}$. Using $c_{\alpha}$ 's we introduce a second normalization:

$$
\begin{aligned}
& E_{\alpha}=c_{\alpha} \widetilde{E}_{\alpha}, \\
& H_{\alpha}=c_{\alpha}^{2} \widetilde{H}_{\alpha} .
\end{aligned}
$$

By (2.1) these vectors satisfy the following relations:

$$
\begin{aligned}
{\left[E_{\alpha}, E_{-\alpha}\right] } & =H_{\alpha}, \\
\tau E_{\alpha} & =-E_{-\alpha}, \\
B\left(E_{\alpha}, E_{-\alpha}\right) & =\frac{2}{\alpha\left(\widetilde{H}_{\alpha}\right)}=\frac{2}{\langle\alpha, \alpha\rangle}, \\
\alpha\left(H_{\alpha}\right) & =2 .
\end{aligned}
$$

If $\mathfrak{c}$ is the center of $\mathfrak{k}$, then there exists an element $Z \in \mathfrak{c}$ such that $(\operatorname{ad} Z)^{2}=-1$ on $\mathfrak{p}^{\mathbb{C}}$ ([Kn], Theorem 7.117). Let $\mathfrak{p}^{+}$be the $(i)$-eigenspace of $a d Z$ and $\mathfrak{p}^{-}$be the $(-i)$-eigenspace of $a d Z$. Then $\mathfrak{p}^{+}$and $\mathfrak{p}^{-}$are Abelian Lie subalgebras invariant under the action of $\mathfrak{k}^{\mathbb{C}}$, 
and $\left[\mathfrak{p}^{+}, \mathfrak{p}^{-}\right] \subset \mathfrak{k}^{\mathbb{C}}([\mathrm{H} 1]$, page 313$)$. Moreover, there is an ordering of $\Delta$ decomposing $Q$ so that $Q=Q^{+} \cup Q^{-}$, and

$$
\begin{aligned}
& \mathfrak{p}^{+}=\sum_{\alpha \in Q^{+}} \mathfrak{g}^{\alpha}=\sum_{\alpha \in Q^{+}} \mathbb{C} E_{\alpha}, \\
& \mathfrak{p}^{-}=\sum_{\alpha \in Q^{+}} \mathfrak{g}^{-\alpha}=\sum_{\alpha \in Q^{+}} \mathbb{C} E_{-\alpha} .
\end{aligned}
$$

Indeed, we can select an ordering on $\Delta$ as follows: for two $\operatorname{roots} \alpha, \beta$ we say that $\alpha$ is bigger than $\beta$ if and only if $-i(\alpha-\beta)(Z)>0 . Q^{+}$is referred as the set of positive noncompact roots, while $Q^{-}$is called the set of negative noncompact roots.

For $\alpha \in Q^{+}$, let

$$
\begin{aligned}
X_{\alpha} & =E_{\alpha}+E_{-\alpha}, \\
Y_{\alpha} & =i\left(E_{\alpha}-E_{-\alpha}\right) .
\end{aligned}
$$

Then the set $\left\{X_{\alpha}, Y_{\alpha}\right\}_{\alpha \in Q^{+}}$spans $\mathfrak{p}$.

The restriction of $a d Z$ to $\mathfrak{p}$ gives the complex structure on $\mathfrak{p}$, which will be denote by $\mathcal{J}$. Thus we have

$$
\begin{aligned}
\mathcal{J} X_{\alpha} & =Y_{\alpha}, \\
\mathcal{J} Y_{\alpha} & =-X_{\alpha}, \\
E_{\alpha} & =\frac{1}{2}\left(X_{\alpha}-i Y_{\alpha}\right), \\
E_{-\alpha} & =\frac{1}{2}\left(X_{\alpha}+i Y_{\alpha}\right) .
\end{aligned}
$$

Similarly to (2.7), for every positive compact root $\alpha$, take $X_{\alpha}, Y_{\alpha} \in \mathfrak{k}$ to be

$$
\begin{aligned}
X_{\alpha} & =E_{\alpha}-E_{-\alpha}, \\
Y_{\alpha} & =i\left(E_{\alpha}+E_{-\alpha}\right) .
\end{aligned}
$$

2.2. Some algebraic preliminaries. In this subsection we are going to introduce some further algebraic properties of $\mathfrak{g}^{\mathbb{C}}$, which will be needed later.

First our goal is to describe the restricted root system for $G / K$. Two roots $\alpha, \beta \in \Delta$ are called strongly orthogonal if neither $\alpha+\beta$ nor $\alpha-\beta$ are roots. One can easily check that strong orthogonality implies orthogonality with respect to the form (2.2). Let

$$
\Gamma=\left\{\gamma_{1}, \ldots, \gamma_{r}\right\} \subset Q^{+}
$$

( $r=\operatorname{rank} G / K)$ be a maximal set of strongly orthogonal positive noncompact roots. Then

$$
\mathfrak{a}=\sum_{\gamma \in \Gamma} \mathbb{R} X_{\gamma}
$$

is a maximal Abelian subalgebra of $\mathfrak{p}$. (For a construction of $\Gamma$ we refer to [H1] pages 385-387.) 
Take $\mathfrak{h}^{-}$to be the real span of the elements $i H_{\gamma}$, and $\mathfrak{h}^{+}$to be the orthogonal complement of $\mathfrak{h}^{-}$in $\mathfrak{h}$ via the Killing form $B$ :

$$
\begin{aligned}
\mathfrak{h}^{-} & =\sum_{\gamma \in \Gamma} \mathbb{R} i H_{\gamma}, \\
\mathfrak{h} & =\mathfrak{h}^{-} \oplus_{B} \mathfrak{h}^{+} .
\end{aligned}
$$

Let $\alpha, \beta \in \Delta$. Denote $\alpha \sim \beta$ if and only if $\left.\alpha\right|_{\mathfrak{h}^{-}}=\left.\beta\right|_{\mathfrak{h}^{-}}$. Define:

$$
\begin{array}{ll}
C_{0}=\{\alpha \in C: \alpha \sim 0\}, & \\
C_{i}=\left\{\alpha \in C: \alpha \sim-\frac{1}{2} \gamma_{i}\right\} & \text { for } i=1, \ldots, r, \\
C_{i j}=\left\{\alpha \in C: \alpha \sim \frac{1}{2}\left(\gamma_{j}-\gamma_{i}\right)\right\} & \text { for } 1 \leq i<j \leq r, \\
Q_{i}=\left\{\alpha \in Q: \alpha \sim \frac{1}{2} \gamma_{i}\right\} & \text { for } i=1, \ldots, r, \\
Q_{i j}=\left\{\alpha \in Q: \alpha \sim \frac{1}{2}\left(\gamma_{i}+\gamma_{j}\right)\right\} & \text { for } 1 \leq i<j \leq r .
\end{array}
$$

Then the map $\alpha \mapsto \gamma_{i}+\alpha$ is a bijection of $C_{i}$ onto $Q_{i}$ and $C_{i j}$ onto $Q_{i j}$. It is also known that $\Delta^{+}$is the disjoint union of the sets $C_{0}, C_{i}, C_{i j}, \Gamma, Q_{i}, Q_{i j}$, and $Q^{+}$is the disjoint union of the sets $\Gamma, Q_{i}, Q_{i j}$ ([H3], pages 457-460).

We shall call $G / K$ a tube space if all sets $Q_{i}$ are empty. Otherwise $G / K$ is a nontube space.

We introduce numbers $M_{\alpha, \beta}$, which will be helpful in next sections in computing some brackets relations. For $\alpha, \beta \in \Delta$ define $M_{\alpha, \beta}$ by:

$$
\begin{aligned}
{\left[E_{\alpha}, E_{\beta}\right] } & =M_{\alpha, \beta} E_{\alpha+\beta} & & \text { if } \alpha+\beta \in \Delta, \\
M_{\alpha, \beta} & =0 & & \text { if } \alpha+\beta \notin \Delta .
\end{aligned}
$$

(some properties of these numbers are described in [H1], pages 146-152).

We may assume ([H1], Theorem V.5.5) that

$$
M_{\alpha, \beta}=-M_{-\alpha,-\beta} .
$$

Proposition 2.16. Fix $k$ between 1 and $r$.

a) If $\alpha=\gamma_{j} \in \Gamma$ then $\alpha\left(H_{\gamma_{k}}\right)=2 \delta_{j k}$.

b) If $\alpha \in Q_{k}$ or $\alpha \in Q_{j k}$, and $\beta=\alpha-\gamma_{k}$, then $\alpha\left(H_{\gamma_{k}}\right)=1$ and $\langle\alpha, \alpha\rangle=\langle\beta, \beta\rangle=\left\langle\gamma_{k}, \gamma_{k}\right\rangle$.

c) For the rest of positive roots $\alpha: \alpha\left(H_{\gamma_{k}}\right)=0$.

Proof. a) and c) are obvious. We prove b) for $\alpha \in Q_{j k}$. The second case is similar.

Notice that by (2.2) and (2.4)

$$
\alpha\left(H_{\gamma_{k}}\right)=\frac{\langle\alpha, \alpha\rangle}{\left\langle\gamma_{k}, \gamma_{k}\right\rangle} \gamma_{k}\left(H_{\alpha}\right)
$$

therefore it is enough to compute $\alpha\left(H_{\gamma_{k}}\right)$ and $\gamma_{k}\left(H_{\alpha}\right)$. For this purpose we shall look at corresponding roots series. The $\gamma_{k}$-series containing $\alpha$ is $\left\{\alpha-\gamma_{k}, \alpha\right\}$, because $\alpha-2 \gamma_{k} \sim$ $\frac{\gamma_{j}-3 \gamma_{k}}{2}$, which by the above remark implies that $\alpha-2 \gamma_{k}$ is not a root. If $\alpha-\gamma_{k}$ is a root, then $\alpha+\gamma_{k}$ cannot belong to $\Delta$ ([H3], Lemma V.4.4), as well. Hence $\alpha\left(H_{\gamma_{k}}\right)=1$, 
which is immediate consequence of Theorem V.5.3 in [H3]. Similarly we get $\gamma_{k}\left(H_{\alpha}\right)=1$. Therefore $\langle\alpha, \alpha\rangle=\left\langle\gamma_{k}, \gamma_{k}\right\rangle$.

Corollary 2.18. If $\alpha \in Q_{k}$ or $\alpha \in Q_{j k}, \beta=\alpha-\gamma_{k}$ then

$$
M_{\gamma_{k}, \beta}= \pm 1 \text {. }
$$

Proof. We have just proved that $c_{\alpha}=c_{\beta}$ and then the corollary follows from Lemma 5.V.2 in $[\mathrm{H} 1]$.

\section{IRREDUCible Symmetric Siegel domains}

In this chapter we introduce symmetric Siegel domains and following A. Korányi and J. Wolf $[\mathrm{KW}]$, we describe realization of a Hermitian symmetric space as a Siegel domain.

3.1. Preliminaries on irreducible symmetric cones. Let $\Omega$ be an irreducible symmetric cone in an Euclidean space. Our aim is to describe a solvable group $S_{0}$ acting simply transitively on $\Omega$. We are going to use heavily the language of Jordan algebras so we recall briefly some basic facts which will be needed later. The reader is referred to the book of J. Faraut and A. Koranyi $[\mathrm{FK}]$ for more details.

A finite dimensional algebra $V$ with a scalar product $\langle\cdot, \cdot\rangle$ is an Euclidean Jordan algebra, if for all elements $x, y$ and $z$ in $V$ :

$$
\begin{aligned}
x y & =y x, \\
x\left(x^{2} y\right) & =x^{2}(x y), \\
\langle x y, z\rangle & =\langle y, x z\rangle .
\end{aligned}
$$

We denote by $L(x)$ the self-adjoint endomorphism of $V$ given by the multiplication by $x$, i.e. $L(x) y=x y$. For an irreducible symmetric cone $\Omega$ contained in a linear space $V$ of the same dimension, the space $V$ can be made a simple real Euclidean Jordan algebra with unit element $e$, so that

$$
\Omega=\operatorname{int}\left\{x^{2}: x \in V\right\} .
$$

Let $G_{0}$ be the connected component of the group of all transformations in $G L(V)$ which leave $\Omega$ invariant, and let $\mathfrak{g}_{0}$ be its Lie algebra. Then $\mathfrak{g}_{0}$ is a subspace of the space of endomorphisms of $V$ which contains all $L(x)$ for all $x \in V$, as well as all $x \square y$ for $x, y \in V$, where

$$
x \square y=L(x y)+[L(x), L(y)]
$$

(see $[\mathrm{FK}]$ for these properties).

We fix a Jordan frame $\left\{c_{1}, \ldots, c_{r}\right\}$ in $V$, that is, a complete system of orthogonal primitive idempotents:

$$
\begin{aligned}
c_{i}^{2} & =c_{i}, \\
c_{i} c_{j} & =0 \quad \text { if } i \neq j, \\
c_{1}+\ldots+c_{r} & =e
\end{aligned}
$$


and none of the $c_{1}, \ldots, c_{r}$ is a sum of two non-zero idempotents. Let us recall that the length $r$ is independent of the choice of the Jordan frame. It is called the rank of $V$. To have an example in mind, one may think of the space $V$ of the symmetric $r \times r$ matrices endowed with the symmetrized product of matrices $\frac{1}{2}(x y+y x)$. Then the corresponding cone is the set of symmetric positive definite $r \times r$ matrices, the set of diagonal matrices with all entries equal to 0 except for one equal to 1 being a Jordan frame.

The Peirce decomposition of $V$ related to the Jordan frame $\left\{c_{1}, \ldots, c_{r}\right\}$ ([FK], Theorem IV.2.1) may be written as

$$
V=\bigoplus_{1 \leq i \leq j \leq r} V_{i j}
$$

It is given by the common diagonalization of the self-adjoint endomorphism $L\left(c_{j}\right)$ with respect to their only eigenvalues $0, \frac{1}{2}, 1$. In particular $V_{j j}=\mathbb{R} c_{j}$ is the eigenspace of $L\left(c_{j}\right)$ related to 1 , and, for $i<j, V_{i j}$ is the intersection of the eigenspaces of $L\left(c_{i}\right)$ and $L\left(c_{j}\right)$ related to $\frac{1}{2}$. All $V_{i j}$, for $i<j$, have the same dimension $d$.

For each $i<j$, we fix once for all an orthonormal basis of $V_{i j}$, which we note $\left\{e_{i j}^{\alpha}\right\}$, with $1 \leq \alpha \leq d$. To simplify the notation, we write $e_{i i}^{\alpha}=c_{i}$ ( $\alpha$ taking only the value 1$)$. Then the system $\left\{e_{i j}^{\alpha}\right\}$, for $i \leq j$ and $1 \leq \alpha \leq \operatorname{dim} V_{i j}$, is an orthonormal basis of $V$.

Let us denote by $\mathfrak{a}_{0}$ the Abelian subalgebra of $\mathfrak{g}_{0}$ consisting of elements $H=L(a)$, where

$$
a=\sum_{j=1}^{r} a_{j} c_{j} \in \bigoplus_{i} V_{i i} .
$$

We set $\lambda_{j}$ the linear form on $\mathfrak{a}_{0}$ given by $\lambda_{j}(H)=a_{j}$. It is clear that the Peirce decomposition gives also a simultaneous diagonalization of all $H \in \mathfrak{a}_{0}$, namely

$$
H x=L(a) x=\frac{\lambda_{i}(H)+\lambda_{j}(H)}{2} x, \quad x \in V_{i j} .
$$

Let $A_{0}=\exp \mathfrak{a}_{0}$. Then $A_{0}$ is an Abelian group, and this is the Abelian group in the Iwasawa decomposition of $G_{0}$. We now describe the nilpotent part $N_{0}$. Its Lie algebra $\mathfrak{n}_{0}$ is the space of elements $X \in \mathfrak{g}_{0}$ such that, for all $i \leq j$,

$$
X V_{i j} \subset \bigoplus_{\substack{k \geq l \\(k, l)>(i, j)}} V_{k l},
$$

where the pairs ordered lexicographically. Once $\mathfrak{n}_{0}$ is defined, we define $\mathfrak{s}_{0}$ as the direct sum $\mathfrak{n}_{0} \oplus \mathfrak{a}_{0}$. The groups $S_{0}$ and $N_{0}$ are then obtained by taking the exponentials. It follows from the definition of $\mathfrak{n}_{0}$ that the matrices of elements of $\mathfrak{s}_{0}$ and $S_{0}$, in the orthonormal basis $\left\{e_{i j}^{\alpha}\right\}$, are upper-triangular.

The solvable group $S_{0}$ acts simply transitively on $\Omega$. This may be found in [FK] Chapter VI, as well as the precise description of $\mathfrak{n}_{0}$ which will be needed later. One has

$$
\mathfrak{n}_{0}=\bigoplus_{i<j \leq r} \mathfrak{n}_{i j}
$$


where

$$
\mathfrak{n}_{i j}=\left\{z \square c_{i}: z \in V_{i j}\right\} .
$$

This decomposition corresponds to a diagonalization of the adjoint action of $\mathfrak{a}_{0}$ since

$$
[H, X]=\frac{\lambda_{j}(H)-\lambda_{i}(H)}{2} X, \quad X \in \mathfrak{n}_{i j} .
$$

Finally, let $V^{\mathbb{C}}=V+i V$ be the complexification of $V$. We extend the action of $G$ to $V^{\mathbb{C}}$ in the obvious way.

3.2. Irreducible symmetric Siegel domains. Suppose that we are given a complex vector space $\mathcal{Z}$ and a Hermitian symmetric bilinear mapping

$$
\Phi: \mathcal{Z} \times \mathcal{Z} \mapsto V^{\mathbb{C}} .
$$

We assume that

$$
\begin{aligned}
\Phi(\zeta, \zeta) & \in \bar{\Omega}, \quad \zeta \in \mathcal{Z}, \\
\text { and } \Phi(\zeta, \zeta) & =0 \text { implies } \zeta=0 .
\end{aligned}
$$

The Siegel domain associated with these data is defined as

$$
{ }^{c} \mathcal{D}=\left\{(\zeta, z) \in \mathcal{Z} \times V^{\mathbb{C}}: \Im z-\Phi(\zeta, \zeta) \in \Omega\right\}^{3} .
$$

It is called of tube type, if $\mathcal{Z}$ is reduced to $\{0\}$. Otherwise, it is called of type II.

There is a representation $\sigma: S_{0} \ni s \rightarrow \sigma(s) \in G L(\mathcal{Z})$ such that

$$
s \Phi(\zeta, w)=\Phi(\sigma(s) \zeta, \sigma(s) w),
$$

and such that all automorphisms $\sigma(s)$, for $s \in A_{0}$, admit a joint diagonalization (see $[\mathrm{KW}])$. To reduce notations, we shall as well denote by $\sigma$ the corresponding representation of the algebra $\mathfrak{s}_{0}$. For $X \in \mathfrak{s}_{0},(3.8)$ implies that

$$
X \Phi(\zeta, w)=\Phi(\sigma(X) \zeta, w)+\Phi(\zeta, \sigma(X) w) .
$$

As an easy consequence, one can prove that the only possible eigenvalues for $\sigma(H)$, with $H \in \mathfrak{a}_{0}$ are $\lambda_{j}(H) / 2$, for $j=1, \ldots, r$. So we may write

$$
\mathcal{Z}=\bigoplus_{j=1}^{r} \mathcal{Z}_{j}
$$

with the property that

$$
\sigma(H) \zeta=\frac{\lambda_{j}(H)}{2} \zeta, \quad \zeta \in \mathcal{Z}_{j} .
$$

Moreover, all the spaces $\mathcal{Z}_{j}$ have the same dimension. A proof of these two facts may be found in $[\mathrm{DHMP}]$. We call $\chi$ the dimension of $\mathcal{Z}_{j}$ for $j=1, \ldots, r$. Let us remark, using (3.9) and (3.11), that for $\zeta, w \in \mathcal{Z}_{j}$, we have $L\left(c_{j}\right) \Phi(\zeta, w)=\Phi(\zeta, w)$. Therefore,

\footnotetext{
${ }^{3}$ We denote a Siegel domain by ${ }^{c} \mathcal{D}$ to be consistent with A. Korányi and J. Wolf notation needed in the next subsections.
} 
$\Phi(\zeta, w)=Q_{j}(\zeta, w) c_{j}$, for $\zeta, w \in \mathcal{Z}_{j}$. Moreover, $\left\langle c_{j}, \Phi(\zeta, \zeta)\right\rangle>0$ for $\zeta \in \mathcal{Z}_{j}$ and so the Hermitian form $Q_{j}$ is positive definite on $c z_{j}$.

The representation $\sigma$ allows to consider $S_{0}$ as a group of holomorphic automorphisms of ${ }^{c} \mathcal{D}$. More generally, the elements $\zeta \in \mathcal{Z}, x \in V$ and $s \in S_{0}$ act on ${ }^{c} \mathcal{D}$ in the following way:

$$
\begin{aligned}
& \zeta \cdot(w, z)=(\zeta+w, z+2 i \Phi(w, \zeta)+i \Phi(\zeta, \zeta)), \\
& x \cdot(w, z)=(w, z+x), \\
& s \cdot(w, z)=(\sigma(s) w, s z) .
\end{aligned}
$$

We call $N(\Phi)$ the group corresponding to the first to actions, that is $N(\Phi)=\mathcal{Z} \times V$ with the product

$$
(\zeta, x)\left(\zeta^{\prime}, x^{\prime}\right)=\left(\zeta+\zeta^{\prime}, x+x^{\prime}+2 \Im \Phi\left(\zeta, \zeta^{\prime}\right)\right)
$$

All three actions generate a solvable Lie group

$$
S=N(\Phi) S_{0}=N(\Phi) N_{0} A_{0}=N A_{0},
$$

which identifies with a group of holomorphic automorphisms acting simply transitively on ${ }^{c} \mathcal{D}$. The group $N(\Phi)$, that is two-step nilpotent, is a normal subgroup of $S$. The Lie algebra $\mathfrak{s}$ of $S$ admits the decomposition

$$
\mathfrak{s}=\mathfrak{n}(\Phi) \oplus \mathfrak{s}_{0}=\left(\bigoplus_{1 \leq j \leq r} \mathcal{Z}_{j}\right) \oplus\left(\bigoplus_{1 \leq i \leq j \leq r} V_{i j}\right) \oplus\left(\bigoplus_{1 \leq i<j \leq r} \mathfrak{n}_{i j}\right) \oplus \mathfrak{a}_{0} .
$$

Moreover, by (3.3), (3.6) and (3.11), one knows the adjoint action of elements $H \in \mathfrak{a}_{0}$ :

$$
\begin{array}{ll}
{[H, X]=\frac{\lambda_{j}(H)}{2} X,} & \text { for } X \in \mathcal{Z}_{j}, \\
{[H, X]=\frac{\lambda_{i}(H)+\lambda_{j}(H)}{2} X,} & \text { for } X \in V_{i j}, \\
{[H, X]=\frac{\lambda_{j}(H)-\lambda_{i}(H)}{2} X,} & \text { for } X \in \mathfrak{n}_{i j} .
\end{array}
$$

Since $S$ acts simply transitively on the domain ${ }^{c} \mathcal{D}$, we may identify $S$ and ${ }^{c} \mathcal{D}$. More precisely, we define

$$
\theta: S \ni s \rightarrow \theta(s)=s \cdot \mathbf{e} \in{ }^{c} \mathcal{D},
$$

where $\mathbf{e}$ is the point $(0, i e)$ in ${ }^{c} \mathcal{D}$. The Lie algebra $\mathfrak{s}$ is then identified with the tangent space of ${ }^{c} \mathcal{D}$ at $\mathbf{e}$ using the differential $d \theta_{e}$. We identify $e$ with the unit element of $S$. We then transport both the Bergman metric $g$ and the complex structure $\mathcal{J}$ from ${ }^{c} \mathcal{D}$ to $S$, where they become left-invariant tensor fields on $S$. We still write $\mathcal{J}$ for the complex structure on $S$.

3.3. Realization of Hermitian symmetric space as a Siegel domain. The goal of this subsection is to describe connections between Hermitian symmetric spaces and Siegel domains. For a space $G / K$, which is supposed to be in the Harish-Chandra realization, we find a biholomorphically equivalent domain, equipped with the structure of Siegel 
domains. We follow closely $[\mathrm{KW}]$ and $[\mathrm{K}]$, but at the end we shall need a little more, namely we are interesting in full description of a basis of $\mathfrak{s}$ in terms of the Lie algebra $\mathfrak{g}^{\mathbb{C}}$.

First we recall the Harish-Chandra realization. Let us denote the analytic subgroups of $G^{\mathbb{C}}$ corresponding to subalgebras $\mathfrak{p}^{+}, \mathfrak{p}^{-}$by $P^{+}$and $P^{-}$, respectively. They are Abelian. The exponential map from $\mathfrak{p}^{ \pm}$to $P^{ \pm}$is biholomorphic and $P^{ \pm}$is biholomorphically equivalent with $\mathbb{C}^{n}$ for some $n$.

The mapping $\left(p_{1}, k, p_{2}\right) \mapsto p_{1} k p_{2}$ is a diffeomorphism of $P^{+} \times K^{\mathbb{C}} \times P^{-}$onto an open submanifold of $G^{\mathbb{C}}$ containing $G$. For $g \in G$ let $p_{+}(g)$ denote the unique element in $\mathfrak{p}^{+}$such that $g \in \exp \left(p_{+}(g)\right) K^{\mathbb{C}} P^{-}$. One can show that $p_{+}(g)=p_{+}(g k)$ and $p_{+}$is a diffeomorphism of $G / K$ onto a domain $\mathcal{D} \subset \mathfrak{p}^{+}=\mathbb{C}^{n}$. $G$ acts biholomorphically on $\mathcal{D}$ by $g \cdot p_{+}(\widetilde{g})=p_{+}(g \widetilde{g})$. Let $o=p_{+}(e)$, then $\mathcal{D}$ is the $G$-orbit of $o$ and the group $K$ is the stabilizer of the point $o$. This is the Harish-Chandra embedding and in fact realizes $G / K$ as a bounded symmetric domain (we refer for more details to [H1] or [Kn]).

From now we shall assume that $\mathcal{D}$ is the above realization. Put $X_{\Gamma}=\sum_{\gamma \in \Gamma} X_{\gamma}$, $E_{\Gamma}=\sum_{\gamma \in \Gamma} E_{\gamma}$ and define an element of $G^{\mathbb{C}}$ called the Cayley transform:

$$
c=\exp \frac{\pi}{4} i X_{\Gamma}
$$

Let

$$
\begin{aligned}
{ }^{c} G & =\operatorname{Ad}(c) G, \\
{ }^{c} K & =\operatorname{Ad}(c) K, \\
{ }^{c} \mathfrak{g} & =\operatorname{Ad}(c) \mathfrak{g}, \\
{ }^{c} \mathfrak{k} & =\operatorname{Ad}(c) \mathfrak{k} .
\end{aligned}
$$

For $g \in G, c \exp \left(p_{+}(g)\right) \in P^{+} K^{c} P^{-}$and so the mapping $p_{+}(g) \mapsto p_{+}\left(c \exp p_{+}(g)\right)$ defines a biholomorphism of $\mathcal{D}$ onto a domain ${ }^{c} \mathcal{D} \subset \mathfrak{p}^{+}([\mathrm{KW}])$. Clearly, ${ }^{c} \mathcal{D}$ is the orbit of the point $c \cdot o=i E_{\gamma}$ under the action of the group ${ }^{c} G$, and ${ }^{c} K$ is the isotropy group of $i E_{\gamma}$.

A simple computation proves the following lemma

Lemma 3.19 ([K], Lemma IV.1.1). Let $\gamma \in \Gamma$. Then

$$
\begin{aligned}
A d(c) \cdot X_{\gamma} & =X_{\gamma}, \\
A d(c) \cdot Y_{\gamma} & =H_{\gamma}, \\
\operatorname{Ad}(c) \cdot H_{\gamma} & =-Y_{\gamma} .
\end{aligned}
$$

Furthermore $A d(c)$ acts trivially on $\mathfrak{h}^{+}$.

It can be shown that $c^{8}=I$ and $A d\left(c^{4}\right)$ preserves $\mathfrak{k}$ and $\mathfrak{p}$. We decompose both Lie algebras:

$$
\begin{aligned}
& -\mathfrak{p}_{T}, \mathfrak{p}_{2} \text { is the }( \pm 1) \text {-eigenspace of } \operatorname{Ad}\left(c^{4}\right) \text { in } \mathfrak{p}, \\
& -\mathfrak{k}_{T}, \mathfrak{q}_{2} \text { is the }( \pm 1) \text {-eigenspace of } \operatorname{Ad}\left(c^{4}\right) \text { in } \mathfrak{k}, \\
& -\mathfrak{g}_{T}=\mathfrak{k}_{T} \oplus \mathfrak{p}_{T} .
\end{aligned}
$$


If $\mathfrak{g}_{T}=\mathfrak{g}$, then the space $G / K$ is of tube type, otherwise it is of nontube type. In the obvious way we introduce Lie algebras $\mathfrak{p}_{T}^{\mathbb{C}}, \mathfrak{p}_{T}^{+}$, etc., and analytic subgroups of $G^{\mathbb{C}}: G_{T}$, $K_{T}$, etc. We denote by $\mathfrak{p}^{j}, \mathfrak{p}^{j k}, \mathfrak{p}^{\frac{j}{2}}$ subspaces corresponding to restricted roots $\gamma_{j}, \frac{\gamma_{j}+\gamma_{k}}{2}$, $\frac{\gamma_{j}}{2}$. Similarly define subspaces of $\mathfrak{k}^{\mathbb{C}}: \mathfrak{k}^{k j}, \mathfrak{k}^{\frac{j}{2}}, \mathfrak{m}^{\mathbb{C}}$ which are restricted root spaces of $\frac{\gamma_{j}-\gamma_{k}}{2}$, $\frac{\gamma_{j}}{2}$ and 0 , respectively. Notice that

$$
\begin{aligned}
& \mathfrak{p}_{T}^{+}=\left(\bigoplus \mathfrak{p}^{j}\right) \oplus\left(\bigoplus_{j<k} \mathfrak{p}^{j k}\right), \\
& \mathfrak{k}_{T}^{\mathbb{C}}=\mathfrak{m}^{\mathbb{C}} \oplus\left(\mathfrak{h}^{-}\right)^{\mathbb{C}} \oplus\left(\bigoplus_{j \neq k} \mathfrak{k}^{j k}\right) .
\end{aligned}
$$

Furthermore,

$$
\begin{aligned}
& \mathfrak{p}_{2}^{+}=\bigoplus^{\frac{j}{2}}, \\
& \mathfrak{q}_{2}^{+}=\bigoplus^{\mathfrak{k}^{\frac{j}{2}}} .
\end{aligned}
$$

Next we introduce

$$
\begin{aligned}
\mathfrak{n}^{j} & ={ }^{c} \mathfrak{g} \cap \mathfrak{p}^{j}, \\
\mathfrak{n}^{j k} & ={ }^{c} \mathfrak{g} \cap \mathfrak{p}^{j k} \quad \text { for } j<k, \\
\mathfrak{n}_{K}^{k j} & ={ }^{c} \mathfrak{g} \cap \mathfrak{k}^{k j} \quad \text { for } j>k, \\
\mathfrak{n}^{\frac{j}{2}} & ={ }^{c} \mathfrak{g} \cap\left(\mathfrak{p}^{\frac{j}{2}} \oplus \mathfrak{k}^{\frac{j}{2}}\right) .
\end{aligned}
$$

Moreover, let

$$
\begin{aligned}
\mathfrak{n}_{T}^{+} & =\left(\bigoplus \mathfrak{n}^{j}\right) \oplus\left(\bigoplus_{j<k} \mathfrak{n}^{j k}\right), \\
\mathfrak{n}_{2}^{+} & =\bigoplus_{j} \mathfrak{n}^{\frac{j}{2}} \\
\mathfrak{n}_{K} & =\bigoplus_{j>k} \mathfrak{n}_{K}^{k j} .
\end{aligned}
$$

One can easily see that $\mathfrak{n}_{T}^{+}={ }^{c} \mathfrak{g} \cap \mathfrak{p}_{T}^{+}$and is a real form of $\mathfrak{p}_{T}^{+}$. Similarly $\mathfrak{n}_{2}^{+}$is a real form of $\mathfrak{q}_{2}^{+} \oplus \mathfrak{p}_{2}^{+}$. One can prove that $A d\left(c^{2}\right)$ preserves $\mathfrak{k}_{T}$ and $\mathfrak{p}_{T}$. We define:

$$
\mathfrak{l}, \mathfrak{q}_{T} \text { is the }( \pm 1) \text {-eigenspace of } A d(c) \text { in } \mathfrak{k}_{T} .
$$

Take $\mathfrak{k}_{T}^{*}=\mathfrak{l} \oplus i \mathfrak{q}_{T}$, then $\mathfrak{k}_{T}^{*}={ }^{c} \mathfrak{g} \cap \mathfrak{k}_{T}^{\mathbb{C}}\left([\mathrm{K}]\right.$, Lemma IV.2.6). By $K_{T}^{*}$ and $L$ we denote corresponding Lie groups.

Now we are ready to describe the domain ${ }^{c} \mathcal{D}$. The image of the point $i E_{\gamma}$ under the action of $K_{T}^{*}$ is a self dual cone in $i \mathfrak{n}_{T}^{+}$, with the group $L$ as a stabilizer of $i E_{\gamma}([\mathrm{K}]$, 
Theorem IV.2.10). We shall denote this cone by $\Omega$. Define a function $\Phi: \mathfrak{p}_{2}^{+} \times \mathfrak{p}_{2}^{+} \mapsto \mathfrak{p}_{T}^{+}$ by

$$
\Phi(X, Y)=\frac{1}{2} a d X(a d Y)^{*} E_{\Gamma},
$$

where $(a d Y)^{*}$ is the adjoint operator of $a d Y$ with respect to $B_{\tau}$. It can be shown $([\mathrm{KW}]$, lemma 6.4) that $\Phi$ satisfies all asumptions listed in the previous subsection and

$$
{ }^{c} \mathcal{D}={ }^{c} G \cdot i E_{\Gamma}=\left\{X+i Y+Z: X, Y \in \mathfrak{n}_{T}^{+}, Z \in \mathfrak{p}_{2}^{+}, Y-\Phi(Z, Z) \in \Omega\right\} .
$$

([KW], Theorem 6.8). Therefore ${ }^{c} \mathcal{D}$ is a Siegel domain of type I or II.

Now we want to recognize the group $S$ in this picture. The mapping $i \mathfrak{q}_{T} \mapsto \mathfrak{n}_{T}^{+}$given by

$$
i \mathfrak{q}_{T} \ni X \mapsto\left[X, E_{\Gamma}\right]
$$

is a bijection ([KV], lemma 2.5). Let $L$ be the inverse map, then we define multiplication in $\mathfrak{n}_{T}^{+}$by:

$$
X Y=\operatorname{adL}(X) a d L(Y) E_{\Gamma} .
$$

One can easily prove that the above definition coincides with the one given in $[\mathrm{FK}]$, page 49, and multiplication so defined gives the structure of Jordan algebra in $\mathfrak{n}_{T}^{+}$.

Take $c_{i}=E_{\gamma_{i}}$, then $L\left(c_{i}\right)=\frac{1}{2} H_{\gamma_{i}}$ and the set $\left\{c_{1}, \ldots, c_{r}\right\}$ is a Jordan frame. The Peirce decomposition (3.2) with respect to this frame is given by the decomposition (3.20) of $\mathfrak{n}_{T}^{+}$with $V_{j}=\mathfrak{n}^{j}$ and $V_{j k}=\mathfrak{n}^{j k}$. Then $\mathfrak{a}_{0}$ is spanned by vectors $H_{\gamma_{i}}$ and the Gauss decomposition (3.4) of $\mathfrak{n}_{0}=\mathfrak{n}_{K}$ coincides with (3.20) for $\mathfrak{n}_{i j}=\mathfrak{n}_{K}^{i j}$. Therefore, the solvable part of Iwasawa decomposition of ${ }^{c} \mathfrak{g}$ with respect to $\mathfrak{a}_{0}$ is

$$
\mathfrak{s}=\mathfrak{n}_{2}^{+} \oplus \mathfrak{n}_{T}^{+} \oplus \mathfrak{n}_{K} \oplus \mathfrak{a}_{0},
$$

and this is exactly the same decomposition as (3.15). The group $S$ corresponding to $\mathfrak{s}$ acts simply transitively on the domain ${ }^{c} \mathcal{D}$.

3.4. An orthonormal basis for a Siegel domain of type II. Now we describe an orthonormal basis of $\mathfrak{s}$ for the nontube case corresponding to the decomposition (3.15). This will be the same basis as in [DHMP], [DHP], [BBDHPT]. We begin with finding a basis of $\mathfrak{n}^{i j}$. Take $\alpha \in Q_{i j}$ and put

$$
\widetilde{\alpha}=\gamma_{i}+\gamma_{j}-\alpha
$$

then by [L] (page 141) $\widetilde{\alpha} \in Q_{i j}$. The classification theorem for Hermitian symmetric spaces says that in the nontube case dimension of each space $Q_{i j}$ is even. Since $\alpha \neq \frac{\gamma_{i}+\gamma_{j}}{2}$ if and only if $\widetilde{\alpha} \neq \frac{\gamma_{i}+\gamma_{j}}{2}$, it follows that $\alpha \neq \widetilde{\alpha}$. By $\bar{Q}_{i j}$ we shall denote the subset of $Q_{i j}$ such that from each pair of roots $\alpha$ and $\widetilde{\alpha}$ exactly one is contained in $\bar{Q}_{i j}$.

Define

$$
\begin{aligned}
& \beta=\alpha-\gamma_{i}=\gamma_{j}-\widetilde{\alpha} \\
& \theta=\alpha-\gamma_{j}=\gamma_{i}-\widetilde{\alpha}
\end{aligned}
$$


Then $\beta$ and $\theta$ are positive compact roots. Applying Lemma V.5.1 in [H1] and Corollary (2.18) we obtain:

$$
\begin{aligned}
\varepsilon & =M_{\gamma_{i}, \beta}=M_{\beta,-\alpha}=M_{-\alpha, \gamma_{i}}=-M_{-\beta, \alpha}=-M_{\alpha,-\gamma_{i}}=-M_{-\gamma_{i},-\beta}, \\
\delta & =M_{\gamma_{j}, \theta}=M_{\theta,-\alpha}=M_{-\alpha, \gamma_{j}}=-M_{-\theta, \alpha}=-M_{\alpha,-\gamma_{j}}=-M_{-\gamma_{j},-\theta}, \\
\rho & =M_{-\gamma_{j}, \beta}=M_{\beta, \widetilde{\alpha}}=M_{\widetilde{\alpha},-\gamma_{j}}=-M_{-\beta,-\widetilde{\alpha}}=-M_{-\widetilde{\alpha}, \gamma_{j}}=-M_{\gamma_{j},-\beta}, \\
\sigma & =M_{-\gamma_{i}, \theta}=M_{\theta, \widetilde{\alpha}}=M_{\widetilde{\alpha},-\gamma_{i}}=-M_{-\theta,-\widetilde{\alpha}}=-M_{-\widetilde{\alpha}, \gamma_{i}}=-M_{\gamma_{i},-\theta},
\end{aligned}
$$

for some $\varepsilon, \sigma, \rho, \delta \in\{-1,1\}$. We have $\alpha+\widetilde{\alpha}-\gamma_{i}-\gamma_{j}=0$, thus it follows from Lemma V.5.3 [H1] that $\varepsilon \rho=\delta \sigma$, which implies

$$
\varepsilon \delta=\left(\delta \sigma \rho^{-1}\right) \delta=\delta^{2} \sigma \rho^{-1}=\sigma \rho .
$$

From (3.18) and (3.24) we obtain

$$
\begin{aligned}
A d\left(c^{-1}\right) E_{\alpha} & =\frac{1}{2} E_{\alpha}-\frac{1}{2} \varepsilon \rho E_{-\widetilde{\alpha}}-\frac{1}{2} i\left(\varepsilon E_{\beta}+\delta E_{\theta}\right), \\
A d\left(c^{-1}\right) E_{\widetilde{\alpha}} & =\frac{1}{2} E_{\widetilde{\alpha}}-\frac{1}{2} \varepsilon \rho E_{-\alpha}+\frac{1}{2} i\left(\sigma E_{-\theta}+\rho E_{-\beta}\right),
\end{aligned}
$$

therefore $A d\left(c^{-1}\right)\left(E_{\alpha}-\varepsilon \rho E_{\widetilde{\alpha}}\right)$ and $A d\left(c^{-1}\right) \cdot i\left(E_{\alpha}+\varepsilon \rho E_{\widetilde{\alpha}}\right)$ are elements of $\mathfrak{g}$, hence $A_{\alpha}=$ $E_{\alpha}-\varepsilon \rho E_{\widetilde{\alpha}}, B_{\alpha}=i\left(E_{\alpha}+\varepsilon \rho E_{\widetilde{\alpha}}\right)$ belong to $\mathfrak{n}^{i j}$. Calculating dimensions we see that the vectors having the above form are a basis of $\mathfrak{n}^{i j}$. To write a basis of $\mathfrak{n}_{i j}$ we shall use the formula (3.5) and compute $C_{\alpha}=2 A_{\alpha} \square c_{i}$ and $D_{\alpha}=2 B_{\alpha} \square c_{i}$ (see (3.1)). Take

$$
X=\frac{1}{2}\left(-\delta\left(E_{\theta}+E_{-\theta}\right)-\varepsilon\left(E_{\beta}+E_{-\beta}\right)\right),
$$

then $\left[X, E_{\Gamma}\right]=A_{\alpha}$, therefore $L\left(A_{\alpha}\right)=X$, and

$$
\begin{aligned}
C_{\alpha} & =2\left(\left[L\left(A_{\alpha}\right), L\left(c_{i}\right)\right]+L\left(A_{\alpha} c_{i}\right)\right) \\
& =\left[X, H_{\gamma_{i}}\right]+X \\
& \left.=\frac{1}{2}\left(\delta E_{\theta}-\delta E_{-\theta}-\varepsilon E_{\beta}+\varepsilon E_{-\beta}\right)\right)+\frac{1}{2}\left(-\delta\left(E_{\theta}+E_{-\theta}\right)-\varepsilon\left(E_{\beta}+E_{-\beta}\right)\right) \\
& =-\delta E_{-\theta}-\varepsilon E_{\beta} .
\end{aligned}
$$

Similarly we can compute:

$$
D_{\alpha}=i\left(\delta E_{-\theta}-\varepsilon E_{\beta}\right) .
$$

Our last step it to write a basis of $\mathfrak{n}_{2}^{+}$. By [KW], Lemma 6.5, the map $\psi=I+A d\left(c^{2}\right) \tau$ is a real linear isomorphism of $\mathfrak{p}_{2}^{+}$onto $\mathfrak{n}_{2}^{+}$. The dimension argument proves that vectors of the form

$$
\psi\left(E_{\alpha}\right), \psi\left(i E_{\alpha}\right)
$$

for $\alpha \in Q_{i}$, are a basis of $\mathfrak{n}_{2}^{+}$.

The vectors

$$
\left\{E_{\gamma_{i}}\right\},\left\{\frac{1}{\sqrt{2}}\left(E_{\alpha}-\varepsilon \rho E_{\widetilde{\alpha}}\right)\right\},\left\{\frac{i}{\sqrt{2}}\left(E_{\alpha}+\varepsilon \rho E_{\widetilde{\alpha}}\right)\right\},\left\{\frac{1}{\sqrt{2}} \psi\left(E_{\alpha}\right)\right\}
$$




$$
\left\{\frac{1}{2} H_{\gamma_{j}}\right\},\left\{\frac{1}{\sqrt{2}}\left(-\delta E_{-\theta}-\varepsilon E_{\beta}\right)\right\},\left\{\frac{i}{\sqrt{2}}\left(\delta E_{-\theta}-\varepsilon E_{\beta}\right)\right\},\left\{\frac{1}{\sqrt{2}} \psi\left(i E_{\alpha}\right)\right\}
$$

form an orthonormal basis of $\mathfrak{s}$ with respect to the Hermitian product $B_{\tau}$. We denote the corresponding left-invariant vector fields on $S$ respectively by:

$$
X_{i}, X_{\alpha}^{1}, X_{\alpha}^{2}, \mathcal{X}_{\alpha}, H_{j}, Y_{\alpha}^{1}, Y_{\alpha}^{2}, \mathcal{Y}_{\alpha},
$$

and we introduce in $\mathfrak{p}^{+}$coordinates corresponding to the basis $X_{i}, X_{\alpha}^{1}, X_{\alpha}^{2}, \mathcal{X}_{\alpha}$ :

$$
w=\sum_{j} w_{j} X_{j}(e)+\sum_{i, j} \sum_{\alpha \in \bar{Q}_{i j}}\left(w_{\alpha}^{1} X_{\alpha}^{1}(e)+w_{\alpha}^{2} X_{\alpha}^{2}(e)\right)+\sum_{i} \sum_{\alpha \in Q_{i}} w_{\alpha} \psi^{-1}\left(\mathcal{X}_{\alpha}(e)\right),
$$

Given a function $f$ on ${ }^{c} \mathcal{D}$ let

$$
\tilde{f}(s)=f(s \cdot(c \cdot o)),
$$

then for a left-invariant vector field $W$ on $S$ we have

$$
W \widetilde{f}(s)=\left.\frac{\partial}{\partial t} f(s \exp t W \cdot(c \cdot o))\right|_{t=0}
$$

and so

$$
\begin{aligned}
& X_{j} \widetilde{f}(e)=\partial u_{j} f(c \cdot o), \\
& X_{\alpha}^{k} \widetilde{f}(e)=\partial u_{\alpha}^{k} f(c \cdot o), \\
& \mathcal{X}_{\alpha} \widetilde{f}(e)=\partial u_{\alpha} f(c \cdot o), \\
& H_{j} \widetilde{f}(e)=\partial v_{j} f(c \cdot o), \\
& Y_{\alpha}^{1} \widetilde{f}(e)=\partial v_{\alpha}^{k} f(c \cdot o), \\
& \mathcal{Y}_{\alpha} \widetilde{f}(e)=\partial v_{\alpha} f(c \cdot o),
\end{aligned}
$$

where $w=u+i v$. Therefore the complex structure $\mathcal{J}$ on $\mathfrak{s}$, transported from ${ }^{c} \mathcal{D}$ is:

$$
\begin{aligned}
\mathcal{J}\left(X_{j}\right) & =H_{j}, \\
\mathcal{J}\left(H_{j}\right) & =-X_{j}, \\
\mathcal{J}\left(X_{\alpha}^{k}\right) & =Y_{\alpha}^{k} \\
\mathcal{J}\left(Y_{\alpha}^{k}\right) & =-X_{\alpha}^{k} \\
\mathcal{J}\left(\mathcal{X}_{\alpha}\right) & =\mathcal{Y}_{\alpha} \\
\mathcal{J}\left(\mathcal{Y}_{\alpha}\right) & =-\mathcal{X}_{\alpha} .
\end{aligned}
$$

Finally,

$$
\begin{aligned}
Z_{j} & =X_{j}-i H_{j}, \\
Z_{\alpha}^{k} & =X_{\alpha}^{k}-i Y_{\alpha}^{k}, \\
\mathcal{Z}_{\alpha} & =\mathcal{X}_{\alpha}-i \mathcal{Y}_{\alpha}
\end{aligned}
$$

are holomorphic vector fields. 
Let $Z$ be one of the vectors fields $Z_{j}, Z_{\alpha}^{k}, \mathcal{Z}_{\alpha}, w$ the corresponding coordinate $w_{j}, w_{\alpha}^{k}$ or $w_{\alpha}$ and let $\Delta_{Z}$ be the unique left-invariant differential operator with the property

$$
\Delta_{Z} \tilde{f}(e)=\partial_{w} \partial_{\bar{w}} f(c \cdot o) .
$$

$\Delta_{Z}$ is real, second order, elliptic degenerate and annihilates holomorphic (consequently pluriharmonic) functions and any left-invariant operator with the above properties is a linear combination of such. Therefore $\Delta_{Z}$ 's are building blocks for admissible operators. $\Delta_{Z}$ can be explicitly computed on the whole group $S$ :

$$
\Delta_{Z}=Z \bar{Z}-\nabla_{Z} \bar{Z}=X^{2}+(\mathcal{J} X)^{2}-\nabla_{X} X-\nabla_{\mathcal{J} X} \mathcal{J} X
$$

where $\nabla$ denotes the Riemannian connection on $S$ (see [DHP], [DHMP]).

\section{The Hua system of SECOND ORDER operators AND THE MAIN THEOREM}

Let $\left\{E_{j}\right\}$ be any orthonormal basis of $\mathfrak{p}^{+}$and $\left\{E_{j}^{*}\right\}$ be a dual basis of $\mathfrak{p}^{-}$with respect to the Killing form $B$ of $\mathfrak{g}^{\mathbb{C}}$ (for example $\left\{\widetilde{E}_{\alpha}\right\}_{\alpha \in Q^{+}}$and $\left\{\widetilde{E}_{-\alpha}\right\}_{\alpha \in Q^{+}}$are such bases). Then the Hua system is

$$
\mathcal{H}=\sum_{j, k} E_{j} E_{k}^{*} \otimes\left[E_{k}, E_{j}^{*}\right]
$$

The above definition was given by K. Johnson and A. Koranyi in [JK]. It is clearly an element of $\mathcal{U}^{\mathbb{C}} \otimes \mathfrak{k}^{\mathbb{C}}$, where $\mathcal{U}^{\mathbb{C}}$ is the complexification of the enveloping algebra of $\mathfrak{g}$. One can easily check that $\mathcal{H}$ does not depend on the chosen basis. For this reason we shall write always the operator $\mathcal{H}$ in terms of the base vectors $\left\{\widetilde{E}_{\alpha}\right\}_{\alpha \in Q^{+}}$. We say that a function $f$ defined on $\mathcal{D}$ is Hua-harmonic, if the corresponding function $\tilde{f}$ on $G(\tilde{f}(g)=f(g \cdot o))$ is annihilated by the Hua system. Analogously $f$ is annihilated by a left-invariant operator $U$ on $G$ if $U \widetilde{f}=0$.

Now we are ready to formulate the main result of this paper:

The Main Theorem. Let $\mathcal{D}=G / K$ be an irreducible Hermitian symmetric domain of nontube type and let $f$ be a real function on $\mathcal{D}$. Then $f$ is Hua-harmonic if and only if $f$ is pluriharmonic.

Let us recall that $f$ defined on $\mathcal{D} \subset \mathbb{C}^{n}$ is pluriharmonic if it is the real part of a holomorphic function. Pluriharmonicity is equivalent to being annihilated by all operators $\partial z_{j} \partial \bar{z}_{k}(1 \leq j, k \leq n)$. Since $E_{j} E_{k}^{*} \widetilde{f}(e)=\partial z_{j} \partial \bar{z}_{k} f(0)$ (see [JK], formula 3.18), the Hua system annihilates pluriharmonic functions. For this reason we have only to prove that any Hua-harmonic function is pluriharmonic.

In fact we shall not use the whole Hua system, but only a part of it. More precisely for any basis $\left\{v_{k}\right\}$ of $\mathfrak{k}^{\mathbb{C}}$ define elements $U_{v_{k}}$ of $\mathcal{U}^{\mathbb{C}}$ by

$$
U_{v_{k}}=\sum_{\alpha \in Q^{+}}\left[v_{k}, \widetilde{E}_{\alpha}\right] \widetilde{E}_{\alpha}^{*} .
$$

Then we have a simple proposition 
Proposition $4.3([\mathrm{JK}],[\mathrm{L}])$. Let $f$ be a function on $G$. Then $\mathcal{H} f=0$ if and only if for every $k: U_{v_{k}} f=0$.

By $[\mathrm{JK}]$ and $[\mathrm{L}]$ the Laplace-Beltrami operator is a linear combination of operators $U_{v_{k}}$. Next we define second order differential operators $U_{k}$, which will be called strongly diagonal Hua operators:

$$
U_{k}=\sum_{\alpha \in Q^{+}}\left[\widetilde{H}_{\gamma_{k}}, \widetilde{E}_{\alpha}\right] \widetilde{E}_{\alpha}^{*}
$$

Observe, that in view of proposition (2.16):

$$
\begin{aligned}
U_{k} & =\sum_{\alpha \in Q^{+}} \alpha\left(\widetilde{H}_{\gamma_{k}}\right) \widetilde{E}_{\alpha} \widetilde{E}_{\alpha}^{*} \\
& =\sum_{\alpha \in Q^{+}} \frac{\langle\alpha, \alpha\rangle}{2} \alpha\left(\tilde{H}_{\gamma_{k}}\right) E_{\alpha} E_{-\alpha}=\sum_{\alpha \in Q^{+}} \frac{\langle\alpha, \alpha\rangle}{\left\langle\gamma_{k}, \gamma_{k}\right\rangle} \alpha\left(H_{\gamma_{k}}\right) E_{\alpha} E_{-\alpha} \\
& =2 E_{\gamma_{k}} E_{-\gamma_{k}}+\sum_{\alpha \in Q_{k}} E_{\alpha} E_{-\alpha}+\sum_{j} \sum_{\alpha \in Q_{j k}} E_{\alpha} E_{-\alpha} .
\end{aligned}
$$

To prove pluriharmonicity of any Hua-harmonic function, we shall use only the Laplace Beltrami operator and strongly diagonal Hua operators.

Now we explain the strategy of the proof. Using the classical result of Harish-Chandra [HC] we may expand $f$ in terms of its projections on the spaces of $K$-finite vectors of type $\pi$ :

$$
f=\sum_{\pi \in \widehat{K}} \chi_{\pi} *_{K} f
$$

where $\widehat{K}$ is the set of equivalence classes of irreducible unitary representations of $K$ and $\chi_{\pi}$ is the character of $\pi$. Now $f_{\pi}=\chi_{\pi} *_{K} f$ are $K$-finite functions and are clearly Huaharmonic. In the next section we prove (Theorem (5.1)) that every Hua-harmonic function is $G$-harmonic (i.e. annihilated by all $G$ invariant operators on $G / K$ ), therefore $f_{\pi}$ are $G$-harmonic. Each $K$-finite, $G$-harmonic function is Poisson integral of a continuous bounded function defined on the maximal Furstenberg boundary ([H3], Theorem V.6.1), therefore each $f_{\pi}$ is bounded, and it is enough to prove the main theorem for bounded functions.

Having the growth assumption (which is invariant on biholomorphic mappings), we transfer our problem to the group $S$ acting simply transitively on the Siegel domain ${ }^{c} \mathcal{D}$, and using techniques of $[\mathrm{BDH}]$, we obtain the result. The details are contained in sections 6 and 7 . 


\section{G-HARMONIC FUNCTIONS}

The aim of this section is to prove the following theorem

Theorem 5.1. If $f$ is a Hua-harmonic function, then $f$ is G-harmonic.

The above theorem was proved in the tube case by $[\mathrm{JK}]$ and $[\mathrm{L}]$. Furthermore the authors of the first cited paper remarked that this result holds also for nontube domains, but they didn't give a proof. Our proof follows closely the argument of [L] and finally we get a system of equations that differs only by constants from the one considered by Lassalle. The main step is to prove the following theorem

Theorem 5.2. If $\Phi$ is K-biinvariant, Hua-harmonic function on $G$, then $\Phi$ is constant.

Using this result we can easily prove theorem (5.1):

Proof of theorem (5.1). As we noticed in the previous section, among the Hua operators there is the Laplace-Beltrami operator. Hence $f$ is an analytic function. Take $d k$ to be unimodular normalized Haar measure on $K$. For fixed $g \in G$ define a function on $G$

$$
\Phi(h)=\int_{K} f(g k h K) d k .
$$

Then $\Phi$ is $K$-biinvariant and Hua-harmonic, therefore by theorem (5.2) $\Phi$ is constant. Hence

$$
f(g K)=\int f(g K) d k=\int f(g k K) d k=\Phi(e)=\Phi(h)=\int f(g k h K) d k
$$

and using the Godement theorem ([H2], page 403) we deduce that $f$ is $G$-harmonic.

We are interested in studying how the Laplace - Beltrami operator and strongly diagonal Hua operators act on a $K$-biinvariant function $f$, defined on $G$. From $K A K$ decomposition of $G$ follows that $f$ depends only on $A$. Hence its enough to compute radial parts of $U_{k}$, which we shall denote by $\Delta\left(U_{k}\right) \in \mathcal{U}\left(\mathfrak{a}^{\mathbb{C}}\right)$. For this we determine $\Delta\left(E_{\alpha} E_{-\alpha}\right)$ for all positive noncompact roots $\alpha$, considering three cases when $\alpha$ belongs to $\Gamma, Q_{j}$ or $Q_{i j}$. The main tool to compute radial parts of these operators will be the following proposition:

Proposition $5.3([\mathrm{~L}])$. Let $f \in C^{2}(G)$ be a right $K$-invariant function and let $X \in \mathfrak{k}$. If

$$
f(a \cdot \exp t(X+Y))=f(a)
$$

for all $t$ and some $Y \in \mathfrak{g}$ then

$$
Y^{2} f(a)=([Y, X] f)(a) .
$$

Using (2.8) we have

$$
E_{\alpha} E_{-\alpha}=\frac{1}{4}\left(X_{\alpha}-i Y_{\alpha}\right)\left(X_{\alpha}+i Y_{\alpha}\right)=\frac{1}{4}\left(X_{\alpha}^{2}+Y_{\alpha}^{2}+i\left[X_{\alpha}, Y_{\alpha}\right]\right)=\frac{1}{4}\left(X_{\alpha}^{2}+Y_{\alpha}^{2}\right) \quad \bmod i \mathfrak{k}
$$

Therefore it is enough in each case to compute $\Delta\left(X_{\alpha}^{2}\right)$ and $\Delta\left(Y_{\alpha}^{2}\right)$. Define

$$
\mathfrak{a}^{\prime}=\left\{X=\sum t_{k} X_{\gamma_{k}}: t_{k} \neq 0 ; t_{i} \pm t_{j} \neq 0 \quad \text { for } i \neq j\right\}
$$

and put $A^{\prime}=\exp \mathfrak{a}^{\prime}$. 
Proposition $5.4([\mathrm{~L}])$. The radial part of $E_{\gamma_{k}} E_{-\gamma_{k}}$ on $A^{\prime}$ is equal

$$
\frac{1}{4}\left(X_{\gamma_{k}}^{2}+2 \operatorname{coth} 2 t_{k} X_{\gamma_{k}}\right)
$$

Proof. Take $a \in A^{\prime}$, then

$$
f(a)=f\left(\exp i t H_{\gamma_{k}} \cdot a\right)=f\left(a \cdot A d a^{-1}\left(\exp i t H_{\gamma_{k}}\right)\right)=f\left(a \exp A d a^{-1}\left(i t H_{\gamma_{k}}\right)\right)
$$

Applying commutation relations:

$$
\begin{aligned}
{\left[X_{\gamma_{k}}, i H_{\gamma_{k}}\right] } & =-2 Y_{\gamma_{k}}, \\
{\left[X_{\gamma_{k}}, Y_{\gamma_{k}}\right] } & =-2 i H_{\gamma_{k}},
\end{aligned}
$$

we obtain

$$
\begin{aligned}
A d a^{-1} \cdot i H_{\gamma_{k}} & =A d\left(\exp -t_{k} X_{\gamma_{k}}\right) \cdot i H_{\gamma_{k}} \\
& =\exp a d\left(-t_{k} X_{\gamma_{k}}\right) \cdot i H_{\gamma_{k}}=\cosh \left(2 t_{k}\right) i H_{\gamma_{k}}+\sinh \left(2 t_{k}\right) Y_{\gamma_{k}} .
\end{aligned}
$$

Hence by proposition (5.3)

$$
\left(\sinh \left(2 t_{k}\right) Y_{\gamma_{k}}\right)^{2} f(a)=\left[\sinh \left(2 t_{k}\right) Y_{\gamma_{k}}, \cosh \left(2 t_{k}\right) i H_{\gamma_{k}}\right] f(a) .
$$

And finally by $\left[Y_{\gamma_{k}}, i H_{\gamma_{k}}\right]=2 X_{\gamma_{k}}$, we conclude

$$
Y_{\gamma_{k}}^{2} f(a)=2 \operatorname{coth}\left(2 t_{k}\right) X_{\gamma_{k}} f(a) .
$$

Next we compute $\Delta\left(E_{\alpha} E_{-\alpha}\right)$, for $\alpha \in Q_{i}$

Lemma 5.5. Let $\alpha \in Q_{i}$. Then there exists $U \in \mathfrak{k}$ such that

$$
\begin{aligned}
{\left[U, X_{\alpha}\right] } & =\varepsilon X_{\gamma_{i}}, \\
A d a^{-1} \cdot U & =\cosh t_{i} U-\varepsilon \sinh t_{i} X_{\alpha},
\end{aligned}
$$

where $\varepsilon= \pm 1$, and $a \in A^{\prime}$.

Proof. Take $\beta=\alpha-\gamma_{i}$ and $\varepsilon$ as in (3.24). Put $U=X_{\beta}$ (as defined in (2.9)). Then the following relations hold:

$$
\begin{aligned}
{\left[X_{\gamma_{j}}, U\right] } & =\varepsilon \delta_{i j} X_{\alpha}, \\
{\left[X_{\gamma_{i}}, X_{\alpha}\right] } & =\varepsilon U, \\
{\left[U, X_{\alpha}\right] } & =\varepsilon X_{\gamma_{i}} .
\end{aligned}
$$

To compute them we use the definition of numbers $M_{\alpha, \beta}$, and the observation (see the proof of proposition (2.16)) that the sum of some roots can not be a root. For instance $\alpha+\gamma_{i},-\alpha-\gamma_{i} \notin \Delta$, for this reason

$$
\begin{aligned}
{\left[X_{\gamma_{i}}, X_{\alpha}\right] } & =\left[E_{\gamma_{i}}+E_{-\gamma_{i}}, E_{\alpha}+E_{-\alpha}\right]=\left[E_{\gamma_{i}}, E_{\alpha}\right]+\left[E_{\gamma_{i}}, E_{-\alpha}\right]+\left[E_{-\gamma_{i}}, E_{\alpha}\right]+\left[E_{-\gamma_{i}}, E_{-\alpha}\right] \\
& =-\varepsilon E_{-\beta}+\varepsilon E_{\beta}=\varepsilon U .
\end{aligned}
$$


Let $a=\exp \left(\sum_{j=1}^{l} t_{j} X_{\gamma_{j}}\right) \in A^{\prime}$ and define an endomorphism $M$ of $\mathfrak{g}$ by

$$
M=\operatorname{ad}\left(-\sum_{j=1}^{l} t_{j} X_{\gamma_{j}}\right) .
$$

Then by (5.6),

$$
\begin{aligned}
A d a^{-1} \cdot U & =e^{a d\left(-\sum t_{j} X_{\gamma_{j}}\right)} U=e^{M} U \\
M^{2 n} U & =t_{i}^{2 n} U \\
M^{2 n+1} U & =-\varepsilon t_{i}^{2 n+1} X_{\alpha}
\end{aligned}
$$

and we get

$$
\begin{aligned}
A d a^{-1} \cdot U & =e^{M} U=\left(\sum_{k=0}^{\infty} \frac{t_{i}^{2 k}}{(2 k) !}\right) U-\varepsilon\left(\sum_{k=0}^{\infty} \frac{t_{i}^{2 k+1}}{(2 k+1) !}\right) X_{\alpha} \\
& =\cosh t_{i} U-\varepsilon \sinh t_{i} X_{\alpha}
\end{aligned}
$$

Lemma 5.7. Suppose $\alpha \in Q_{i}$, then there exists $V \in \mathfrak{k}$ such that:

$$
\begin{aligned}
{\left[V, Y_{\alpha}\right] } & =\varepsilon X_{\gamma_{i}}, \\
A d a^{-1} \cdot V & =\cosh t_{i} V-\varepsilon \sinh t_{i} Y_{\alpha},
\end{aligned}
$$

where $\varepsilon$ has the same value like in the previous lemma.

Proof. Taking $V=Y_{\beta}$ (for $\beta$ as in the previous lemma) and repeating the proof we conclude the lemma.

Proposition 5.8. For $\alpha \in Q_{i}$ the radial part of $E_{\alpha} E_{-\alpha}$ on $A^{\prime}$ is:

$$
\frac{1}{2} \operatorname{coth} t_{i} X_{\gamma_{i}} \text {. }
$$

Proof. Using $K$ invariance of the function $f$ and lemma (5.5) we obtain

$$
f(a)=f\left(a \exp \left(\cosh t_{i} U-\varepsilon \sinh t_{i} X_{\alpha}\right)\right) .
$$

Hence by proposition (5.3):

$\left(-\varepsilon \sinh t_{i} X_{\alpha}\right)^{2}=\left[-\varepsilon \sinh t_{i} X_{\alpha}, \cosh t_{i} U\right]=-\varepsilon \sinh t_{i} \cosh t_{i}\left[X_{\alpha}, U\right]=\sinh t_{i} \cosh t_{i} X_{\gamma_{i}}$.

Therefore

Analogously, applying lemma (5.7):

$$
X_{\alpha}^{2}=\operatorname{coth} t_{i} X_{\gamma_{i}}
$$

$$
Y_{\alpha}^{2}=\operatorname{coth} t_{i} X_{\gamma_{i}}
$$

So we have

$$
\Delta\left(E_{\alpha} E_{-\alpha}\right)=\frac{1}{4}\left(\Delta\left(X_{\alpha}^{2}\right)+\Delta\left(Y_{\alpha}^{2}\right)\right)=\frac{1}{4}\left(\operatorname{coth} t_{i} X_{\gamma_{i}}+\operatorname{coth} t_{i} X_{\gamma_{i}}\right)=\frac{1}{2} \operatorname{coth} t_{i} X_{\gamma_{i}} .
$$


The last case is $\alpha \in Q_{i j}$. Then for our purpose it is enough to determinate $\Delta\left(E_{\alpha} E_{-\alpha}+\right.$ $\left.E_{\widetilde{\alpha}} E_{-\widetilde{\alpha}}\right)$, for $\widetilde{\alpha}$ defined by (3.22).

Lemma 5.9. If $\alpha \in Q_{i j}$ then there exist $U, \tilde{U}, V, \tilde{V} \in \mathfrak{k}$ such that

$$
\begin{aligned}
A d a^{-1} U & =\cosh \left(t_{i}+\rho \sigma t_{j}\right) U-\sinh \left(t_{i}+\rho \sigma t_{j}\right)\left(\varepsilon X_{\alpha}+\sigma X_{\widetilde{\alpha}}\right), \\
A d a^{-1} \tilde{U} & =\cosh \left(t_{i}-\rho \sigma t_{j}\right) \tilde{U}-\sinh \left(t_{i}-\rho \sigma t_{j}\right)\left(\varepsilon X_{\alpha}-\sigma X_{\widetilde{\alpha}}\right), \\
A d a^{-1} V & =\cosh \left(t_{i}-\rho \sigma t_{j}\right) V-\sinh \left(t_{i}-\rho \sigma t_{j}\right)\left(\varepsilon Y_{\alpha}+\sigma Y_{\widetilde{\alpha}}\right), \\
A d a^{-1} \tilde{V} & =\cosh \left(t_{i}+\rho \sigma t_{j}\right) V-\sinh \left(t_{i}+\rho \sigma t_{j}\right)\left(\varepsilon Y_{\alpha}-\sigma Y_{\widetilde{\alpha}}\right), \\
{\left[\varepsilon X_{\alpha}+\sigma X_{\widetilde{\alpha}}, U\right] } & =-2\left(X_{\gamma_{i}}+\rho \sigma X_{\gamma_{j}}\right), \\
{\left[\varepsilon X_{\alpha}-\sigma X_{\widetilde{\alpha}}, \tilde{U}\right] } & =-2\left(X_{\gamma_{i}}-\rho \sigma X_{\gamma_{j}}\right), \\
{\left[\varepsilon Y_{\alpha}+\sigma Y_{\widetilde{\alpha}}, V\right] } & =-2\left(X_{\gamma_{i}}-\rho \sigma X_{\gamma_{j}}\right), \\
{\left[\varepsilon Y_{\alpha}-\sigma Y_{\widetilde{\alpha}}, \tilde{V}\right] } & =-2\left(X_{\gamma_{i}}+\rho \sigma X_{\gamma_{j}}\right),
\end{aligned}
$$

where $\varepsilon, \sigma, \rho, \delta \in\{-1,1\}$.

Proof. Take $\beta, \theta, \varepsilon, \delta, \rho, \sigma$, as in (3.23) and (3.24). Next set $U=X_{\beta}+X_{\theta}, \tilde{U}_{\beta}=X_{\beta}-X_{\theta}$, $V=Y_{\beta}+Y_{\theta}, \tilde{V}_{\beta}=Y_{\beta}-Y_{\theta}$. Then applying the methods of the proof of lemma (5.5), and using (3.25) we can easily compute all needed brackets relations, and conclude the lemma.

Proposition 5.10. Let $\alpha \in Q_{i j}$, then on $A^{\prime}$

$$
\Delta\left(E_{\alpha} E_{-\alpha}+E_{\widetilde{\alpha}} E_{-\widetilde{\alpha}}\right)=\frac{1}{2}\left(\operatorname{coth}\left(t_{i}+t_{j}\right)\left(X_{\gamma_{i}}+X_{\gamma_{j}}\right)+\operatorname{coth}\left(t_{i}-t_{j}\right)\left(X_{\gamma_{i}}-X_{\gamma_{j}}\right)\right) .
$$

Proof. By lemma (5.9) we get

$$
f(a)=f\left(a \exp \left(\cosh \left(t_{i}+\rho \sigma t_{j}\right) U-\sinh \left(t_{i}+\rho \sigma t_{j}\right)\left(\varepsilon X_{\alpha}+\sigma X_{\widetilde{\alpha}}\right)\right)\right)
$$

In view of proposition (5.3)

$$
\left(-\sinh \left(t_{i}+\rho \sigma t_{j}\right)\left(\varepsilon X_{\alpha}+\sigma X_{\widetilde{\alpha}}\right)\right)^{2}=-\left[\sinh \left(t_{i}+\rho \sigma t_{j}\right)\left(\varepsilon X_{\alpha}+\sigma X_{\widetilde{\alpha}}\right), \cosh \left(t_{i}+\rho \sigma t_{j}\right) U\right] .
$$

Accordingly

$$
\left(\varepsilon X_{\alpha}+\sigma X_{\widetilde{\alpha}}\right)^{2}=2 \operatorname{coth}\left(t_{i}+\rho \sigma t_{j}\right)\left(X_{\gamma_{i}}+\rho \sigma X_{\gamma_{j}}\right)
$$

and similarly

$$
\left(\varepsilon X_{\alpha}-\sigma X_{\widetilde{\alpha}}\right)^{2}=2 \operatorname{coth}\left(t_{i}-\rho \sigma t_{j}\right)\left(X_{\gamma_{i}}-\rho \sigma X_{\gamma_{j}}\right)
$$

which gives us

$$
\begin{aligned}
2\left(X_{\alpha}^{2}+X_{\tilde{\alpha}}^{2}\right) & =\left(\varepsilon X_{\alpha}+\sigma X_{\widetilde{\alpha}}\right)^{2}+\left(\varepsilon X_{\alpha}-\sigma X_{\widetilde{\alpha}}\right)^{2} \\
& =2\left(\operatorname{coth}\left(t_{i}+t_{j}\right)\left(X_{\gamma_{i}}+X_{\gamma_{j}}\right)+\operatorname{coth}\left(t_{i}-t_{j}\right)\left(X_{\gamma_{i}}-X_{\gamma_{j}}\right)\right) .
\end{aligned}
$$

Analogous computations entail

$$
Y_{\alpha}^{2}+Y_{\widetilde{\alpha}}^{2}=\operatorname{coth}\left(t_{i}+t_{j}\right)\left(X_{\gamma_{i}}+X_{\gamma_{j}}\right)+\operatorname{coth}\left(t_{i}-t_{j}\right)\left(X_{\gamma_{i}}-X_{\gamma_{j}}\right) .
$$


Finally we conclude

$$
\begin{aligned}
\Delta\left(E_{\alpha} E_{-\alpha}+E_{\widetilde{\alpha}} E_{-\widetilde{\alpha}}\right) & =\frac{1}{4}\left(X_{\alpha}^{2}+Y_{\alpha}^{2}+X_{\widetilde{\alpha}}^{2}+Y_{\widetilde{\alpha}}^{2}\right) \\
& =\frac{1}{2}\left(\operatorname{coth}\left(t_{i}+t_{j}\right)\left(X_{\gamma_{i}}+X_{\gamma_{j}}\right)+\operatorname{coth}\left(t_{i}-t_{j}\right)\left(X_{\gamma_{i}}-X_{\gamma_{j}}\right)\right) .
\end{aligned}
$$

Now we are ready to compute radial parts of strongly diagonal Hua operators. Combining propositions (5.4), (5.8), (5.10) and (4.5) we get the radial parts of $U_{k}$ :

$$
\begin{aligned}
2 \Delta\left(U_{k}\right)= & X_{\gamma_{k}}^{2}+2 \operatorname{coth} 2 t_{k} X_{\gamma_{k}}+\beta \operatorname{coth} t_{k} X_{\gamma_{k}} \\
& +\frac{\lambda}{2} \sum_{j \neq k}\left(\operatorname{coth}\left(t_{j}+t_{k}\right)\left(X_{\gamma_{j}}+X_{\gamma_{k}}\right)+\operatorname{coth}\left(t_{j}-t_{k}\right)\left(X_{\gamma_{j}}-X_{\gamma_{k}}\right)\right),
\end{aligned}
$$

where

$$
\begin{aligned}
\beta & =\#\left\{\alpha: \alpha \in Q_{k}\right\}, \\
\lambda & =\#\left\{\alpha: \alpha \in Q_{j k} \text { for some } j \neq k\right\},
\end{aligned}
$$

$\beta$ and $\lambda$ are independent on $j$ and $k$.

Take $f$ to be any $K$-biinvariant analytic function on $G$. Every element $g$ of $G$ we can write (using $K A K$ decomposition) as follows

$$
g=k_{1}\left(\exp \sum_{j=1}^{r} t_{j} X_{\gamma_{j}}\right) k_{2}, \quad k_{1}, k_{2} \in K, \quad t_{j} \in \mathbb{R} .
$$

Therefore $f$ depends only on $t_{j}$ and we can think of it as a function defined on $\mathbb{R}^{r}$. Furthermore $f$ is $W$-invariant, where $W$ denotes the classical Weyl group. $W$ acts on $\mathfrak{a}$ by signed permutations, so

$$
f\left(t_{1}, \ldots, t_{r}\right)=f\left( \pm t_{\sigma(1)}, \ldots, \pm t_{\sigma(r)}\right)
$$

for any permutation $\sigma$ of $r$ elements. Therefore we can write a new version of theorem (5.2) as follows:

Theorem 5.11. Let $\lambda$ and $\beta$ be stricly positive real numbers and let $f$ be an analytic function on $\mathbb{R}^{r}$, invariant under signed permutations, such that $\bar{U}_{k} f=0$ for $k=1, \ldots, l$, where

$$
\begin{aligned}
\bar{U}_{k}= & \partial_{t_{k}}^{2}+2 \operatorname{coth} 2 t_{k} \partial_{t_{k}}+\beta \operatorname{coth} t_{k} \partial_{t_{k}} \\
& +\frac{\lambda}{2} \sum_{j \neq k}\left(\operatorname{coth}\left(t_{j}+t_{k}\right)\left(\partial_{t_{j}}+\partial_{t_{k}}\right)+\operatorname{coth}\left(t_{j}-t_{k}\right)\left(\partial_{t_{j}}-\partial_{t_{k}}\right)\right)
\end{aligned}
$$

Then $f$ is constant.

Introducing new variables $r_{k}=\tanh t_{k}$ we reformulate theorem (5.11) as follows: 
Theorem 5.12. Let $f$ be an analytic function on the cube $\left\{\left(r_{1}, \ldots, r_{r}\right) \in \mathbb{R}^{r},\left|r_{k}\right|<1\right\}$, invariant on signed permutations, and annihilated by the operators

$$
V_{k} f=\left(1-r_{k}^{2}\right)\left(\frac{\partial^{2}}{\partial^{2} r_{k}} f+\frac{1}{r_{k}} \frac{\partial}{\partial r_{k}} f\right)+\frac{\beta}{r_{k}} \frac{\partial}{\partial r_{k}} f+\lambda \sum_{j \neq k} \frac{1-r_{j}^{2}}{r_{k}^{2}-r_{j}^{2}}\left(r_{k} \frac{\partial}{\partial r_{k}} f-r_{j} \frac{\partial}{\partial r_{j}} f\right),
$$

$k=1, \ldots, r$. Then $f$ is constant.

Proof. $f$ is an analytic function invariant on signed permutations, so we can expand it in a series

$$
f\left(r_{1}, \ldots, r_{r}\right)=\sum_{n_{1} \ldots n_{r}} a_{n_{1}, \ldots, n_{r}} r_{1}^{2 n_{1}} \cdot \ldots \cdot r_{r}^{2 n_{r}}
$$

where the coefficients $a_{n_{1}, \ldots, n_{l}}$ are invariant on permutations of indices.

We shall prove by induction with respect to the number of nonzero indices in $a_{n_{1}, \ldots, n_{r}}$, that only $a_{0, \ldots, 0}$ might not be zero.

For the first induction step, take $\bar{f}\left(r_{1}\right)=f\left(r_{1}, \ldots, 0\right)$. Then applying $V_{1}$ we get:

$$
\left(1-r_{1}^{2}\right)\left(\frac{\partial^{2}}{\partial^{2} r_{1}} \bar{f}+\frac{1}{r_{1}} \frac{\partial}{\partial r_{1}} \bar{f}\right)+\frac{\mu}{r_{1}} \frac{\partial}{\partial r_{1}} \bar{f}=0,
$$

where $\mu=(l-1) \lambda+\beta$. Put

$$
g(t)=\bar{f}(t)=\sum b_{n} t^{2 n}
$$

for $b_{n}=a_{n, 0, \ldots, 0}$. Then plugging this function to formula (5.13) we get

$$
\begin{aligned}
0 & =\left(1-t^{2}\right)\left(g^{\prime \prime}(t)+\frac{1}{t} g^{\prime}(t)\right)+\frac{\mu}{t} g^{\prime}(t) \\
& =\sum_{n=0}^{\infty}\left((2 n+2)(2 n+2+\mu) b_{n+1}-(2 n)^{2} b_{n}\right) t^{2 n} .
\end{aligned}
$$

Therefore,

$$
\begin{aligned}
b_{1} & =0, \\
b_{n+1} & =\frac{4 n^{2}}{(2 n+2)(2 n+2+\mu)} b_{n}=0 .
\end{aligned}
$$

Now we would like to prove $a_{n_{1}, \ldots, n_{s}, 0 \ldots 0}=0$ when $\sum n_{i}>0$, using inductive hypothesis for all $p<s$. Define

$$
\bar{f}\left(r_{1}, \ldots, r_{s}\right)=f\left(r_{1}, \ldots, r_{s}, 0, \ldots, 0\right) .
$$

We are going to use $V_{s}$ :

$V_{s} \bar{f}=\left(1-r_{s}^{2}\right)\left(\frac{\partial^{2}}{\partial^{2} r_{s}} \bar{f}+\frac{1}{r_{s}} \frac{\partial}{\partial r_{s}} \bar{f}\right)+\frac{\mu}{r_{s}} \frac{\partial}{\partial r_{s}} \bar{f}+\lambda \sum_{1 \leq j \leq s-1} \frac{1-r_{j}^{2}}{r_{s}^{2}-r_{j}^{2}}\left(r_{s} \frac{\partial}{\partial r_{s}} \bar{f}-r_{j} \frac{\partial}{\partial r_{j}} \bar{f}\right)=0$, 
where $\mu=\lambda(l-s)+\beta$. Because of analycity, the function $\bar{f}$ can be expand in a series

$$
\bar{f}\left(r_{1}, \ldots, r_{s}\right)=\sum_{n_{1}, \ldots, n_{s}} b_{n_{1}, \ldots, n_{s}} r_{1}^{2 n_{1}} \cdot \ldots \cdot r_{s}^{2 n_{s}}
$$

for $b_{n_{1}, \ldots, n_{s}}=a_{n_{1}, \ldots, n_{s}, 0, \ldots, 0}$. In addition, we know that for any permutation $\rho$ of $s$ elements $b_{n_{1}, \ldots, n_{s}}=b_{\rho\left(n_{1}\right), \ldots, \rho\left(n_{s}\right)}$. Hence we obtain

$$
\begin{aligned}
0= & \sum b_{n_{1}, \ldots, n_{s}}\left(\left(\prod_{1 \leq k \leq s-1}\left(r_{s}^{2}-r_{k}^{2}\right)\right) n_{s}\left(\frac{2 n_{s}+\mu}{r_{s}^{2}}-2 n_{s}\right) r_{1}^{2 n_{1}} \cdot \ldots \cdot r_{s}^{2 n_{s}}\right. \\
& \left.+\lambda \sum_{1 \leq j \leq s-1}\left(\prod_{\substack{k \neq j \\
1 \leq k \leq s-1}}\left(r_{s}^{2}-r_{k}^{2}\right)\right)\left(n_{s}-n_{j}\right)\left(1-r_{j}^{2}\right) r_{1}^{2 n_{1}} \cdot \ldots \cdot r_{s}^{2 n_{s}}\right) .
\end{aligned}
$$

First we prove that $b_{n_{1}, \ldots, n_{s-1}, 1}=0$. Choose all factors in which $r_{s}$ does not appear. We have then

$$
\begin{aligned}
0= & \sum b_{n_{1}, \ldots, n_{s-1}, 0}\left(\lambda \sum_{1 \leq j \leq s-1}\left(\prod_{k \neq j} r_{k}^{2}\right) n_{j}\left(1-r_{j}^{2}\right) r_{1}^{2 n_{1}} \cdot \ldots \cdot r_{s-1}^{2 n_{s-1}}\right) \\
& +\sum b_{n_{1}, \ldots, n_{s-1}, 1}(2+\mu) r_{1}^{2\left(n_{1}+1\right)} \cdot \ldots \cdot r_{s-1}^{2\left(n_{s-1}+1\right)} .
\end{aligned}
$$

By our inductive hypothesis all coefficients $b_{n_{1}, \ldots, n_{s-1}, 0}$ are equal to 0 . Therefore due to

$$
\sum b_{n_{1}, \ldots, n_{s-1}, 1} r_{1}^{2\left(n_{1}+1\right)} \cdot \ldots \cdot r_{s-1}^{2\left(n_{s-1}+1\right)}=0,
$$

$b_{n_{1}, \ldots, n_{s-1}, 1}$ must be equal 0 as well.

Suppose that $b_{n_{1}, \ldots, n_{s}}=0$ for $n_{s}<N$ and consider the monomian

$$
r_{1}^{2\left(n_{1}+1\right)} \cdot \ldots \cdot r_{s-1}^{2\left(n_{s-1}+1\right)} r_{s}^{2(N-1)} .
$$

By our assumption the coefficient in front of it is $b_{n_{1}, \ldots, N}$. This implies

$$
b_{n_{1}, \ldots, N}=0,
$$

and completes the induction.

\section{Hua operators on Siegel domains}

Given a function $f$ on $\mathcal{D}$ we define

$$
\begin{aligned}
\tilde{f}(g) & =f(g \cdot o) & & \text { - a function on } G, \\
{ }^{c} f(x) & =f\left(c^{-1} x\right) & & \text { - a function on }{ }^{c} \mathcal{D}, \\
{ }^{c} \widetilde{f}(g) & ={ }^{c} f(g \cdot(c \cdot o)) & & \text { - a function on }{ }^{c} G .
\end{aligned}
$$

To prove that $f$ is pluriharmonic it is enough to show pluriharmonicity of ${ }^{c} f$, because $c$ is biholomorphic.

One can easily check that ${ }^{c} \widetilde{f}(A d(c) g)=\widetilde{f}(g)$ which implies that for any $X \in \mathcal{U}(G)$ :

$$
X \tilde{f}(g)=(A d(c) X)^{c} \tilde{f}(A d(c) g)
$$


where $A d(c) X \in \mathcal{U}\left({ }^{c} G\right)$. Therefore the function ${ }^{c} \tilde{f}$ is annihilated by left invariant operators $A d(c) U_{k}$. Our aim is now to compute these operators on the domain ${ }^{c} \mathcal{D}$ and on the group $S$. As a result we shall get Hua operators on Siegel domain written in terms of admissible operators $\Delta_{Z}$ 's, $Z$ being as in (3.29).

Theorem 6.1. Let $f$ be a Hua-harmonic function on $\mathcal{D}$, then the corresponding function ${ }^{c} f$ on ${ }^{c} \mathcal{D}$ is annihilated by the Laplace-Beltrami operator $\Delta$ on ${ }^{c} \mathcal{D}$ and $\mathcal{H}_{k}(1 \leq k \leq r)$ :

$$
\mathcal{H}_{k}=2 \Delta_{Z_{k}}+\sum_{\alpha \in Q_{k}} \Delta_{\mathcal{Z}_{\alpha}}+\sum_{j} \sum_{\alpha \in \bar{Q}_{k j}}\left(\Delta_{Z_{\alpha}^{1}}+\Delta_{Z_{\alpha}^{2}}\right)
$$

which we shall call strongly diagonal Hua operators as well. The Laplace-Beltrami operator is given by:

$$
\Delta=\sum_{i} \Delta_{Z_{i}}+\sum_{i} \sum_{\alpha \in Q_{i}} \Delta_{\mathcal{Z}_{\alpha}}+\sum_{j, k} \sum_{\alpha \in \bar{Q}_{k j}}\left(\Delta_{Z_{\alpha}^{1}}+\Delta_{Z_{\alpha}^{2}}\right) .
$$

Proof. The idea of the proof is following. First we compute $U_{k}$ 's at the point $o$, denoting the result by $V_{k}$ :

$$
U_{k} \tilde{f}(e)=V_{k} f(o) .
$$

Next using the Cayley transform we find operators ${ }^{c} V_{k}$ such that

$$
{ }^{c} V_{k}{ }^{c} f(c \cdot o)=V_{k} f(o),
$$

and finally we extend them $S$-invariant on the domain ${ }^{c} \mathcal{D}$ and the group $S$.

First observe that by [JK] (3.18)

$$
E_{\alpha} \bar{E}_{\alpha} \widetilde{f}(e)=\partial z_{\alpha} \partial \bar{z}_{\alpha} f(o),
$$

where $\left\{z_{\alpha}\right\}$ are coordinates in $\mathfrak{p}^{+}$with respect to the basis $\left\{E_{\alpha}\right\}_{\alpha \in Q^{+}}$. Therefore

$$
U_{k} \widetilde{f}(e)=V_{k} f(o)=\left(2 \partial z_{k} \partial \bar{z}_{k}+\sum_{\alpha \in Q_{k}} \partial z_{\alpha} \partial \bar{z}_{\alpha}+\sum_{j} \sum_{\alpha \in Q_{j k}} \partial z_{\alpha} \partial \bar{z}_{\alpha}\right) f(o) .
$$

Now we have to compute differential of $c$. We shall use the formula given by [S], Lemma II.5.3, which says that the Jacobian of the mapping $z \mapsto c \cdot z$ at the point $o$ is given by

$$
\operatorname{Jac}(o \mapsto c \cdot o)=\left.A d c_{K}\right|_{\mathfrak{p}^{+}},
$$

where $c_{K}$ denotes the component $K^{\mathbb{C}}$ of $c$ in the decomposition $P^{+} K^{\mathbb{C}} P^{-}$. By $[\mathrm{KW}]$, Lemma 3.5:

$$
c_{K}=\exp \left(-\sum_{1 \leq i \leq r} \log \cosh \left(\frac{\pi}{4} i\right) \cdot H_{\gamma_{i}}\right)=\prod_{1 \leq i \leq r} \exp \left(-\log \frac{\sqrt{2}}{2} \cdot H_{\gamma_{i}}\right) .
$$

Hence the vectors $E_{\alpha}$ are eigenvectors of $\operatorname{Ad}\left(c_{K}\right)$ :

$$
A d c_{K}\left(E_{\alpha}\right)=\left\{\begin{array}{cl}
2 E_{\alpha}, & \text { for } \alpha \in \Gamma \\
2 E_{\alpha}, & \text { for } \alpha \in Q_{i j}, \\
\sqrt{2} E_{\alpha}, & \text { for } \alpha \in Q_{j} .
\end{array}\right.
$$


Therefore,

$$
\begin{aligned}
V_{k} f(o) & =\left(2 \partial z_{k} \partial \bar{z}_{k}+\sum_{\alpha \in Q_{k}} \partial z_{\alpha} \partial \bar{z}_{\alpha}+\sum_{j} \sum_{\alpha \in Q_{j k}} \partial z_{\alpha} \partial \bar{z}_{\alpha}\right) f(o) \\
& =\left(8 \partial z_{k} \partial \bar{z}_{k}+2 \sum_{\alpha \in Q_{k}} \partial z_{\alpha} \partial \bar{z}_{\alpha}+4 \sum_{j} \sum_{\alpha \in Q_{j k}} \partial z_{\alpha} \partial \bar{z}_{\alpha}\right)^{c} f(c \cdot o) \\
& ={ }^{c} V_{k}{ }^{c} f(c \cdot o) .
\end{aligned}
$$

Let us observe that for $\alpha \in Q_{i j}$ and $\widetilde{\alpha}=\gamma_{i}+\gamma_{j}-\alpha$

$$
\begin{aligned}
\left(\partial z_{\alpha} \partial \bar{z}_{\alpha}+\partial z_{\widetilde{\alpha}} \partial \bar{z}_{\widetilde{\alpha}}\right)^{c} f(c \cdot o)= & \left(\partial^{2} x_{\alpha}+\partial^{2} y_{\alpha}+\partial^{2} x_{\widetilde{\alpha}}+\partial^{2} y_{\widetilde{\alpha}}\right)^{c} f(c \cdot o) \\
= & \left(\frac{1}{2}\left(\partial x_{\alpha}-\varepsilon \rho \partial x_{\widetilde{\alpha}}\right)^{2}+\frac{1}{2}\left(\partial y_{\alpha}-\varepsilon \rho \partial y_{\widetilde{\alpha}}\right)^{2}\right. \\
& \left.+\frac{1}{2}\left(\partial x_{\alpha}+\varepsilon \rho \partial x_{\widetilde{\alpha}}\right)^{2}+\frac{1}{2}\left(\partial y_{\alpha}+\varepsilon \rho \partial y_{\widetilde{\alpha}}\right)^{2}\right)^{c} f(c \cdot o) \\
= & \left(\partial w_{\alpha}^{1} \partial \bar{w}_{\alpha}^{1}+\partial w_{\alpha}^{2} \partial \bar{w}_{\alpha}^{2}\right)^{c} f(c \cdot o)
\end{aligned}
$$

for $\varepsilon, \rho$ defined by (3.24) and $w$ as in (3.26). Then applying (3.27) and the definition of building blocks we obtain the $S$-invariant extension of the above operator which is $\Delta_{Z_{\alpha}^{1}}+\Delta_{Z_{\alpha}^{2}}$. In the same way we get the corresponding operators for $\partial z_{i} \partial \bar{z}_{i}$ and $\partial z_{\alpha} \partial \bar{z}_{\alpha}$ (for $\left.\alpha \in Q_{i}\right): \Delta_{Z_{i}}, 2 \mathcal{L}_{Z_{\alpha}}$. Therefore:

$$
0=8 \Delta_{Z_{k}}+4 \sum_{\alpha \in Q_{k}} \Delta_{\mathcal{Z}_{\alpha}}+4 \sum_{j} \sum_{\alpha \in \bar{Q}_{k j}}\left(\Delta_{Z_{\alpha}^{1}}+\Delta_{Z_{\alpha}^{2}}\right)=4 \mathcal{H}_{k}{ }^{c} f(s) .
$$

Observe that using the same method we can transfer the Laplace-Beltrami operator:

$$
\sum_{\alpha \in Q^{+}} E_{\alpha} \overline{E_{\alpha}}
$$

and we get formula (6.3).

Note that to obtain the main result it is enough to prove the following:

Theorem 6.4. Let $f$ be a real, bounded function on $S$, annihilated by the operators:

$$
\Delta, \mathcal{H}_{1}, \ldots, \mathcal{H}_{r}
$$

Then $f$ is pluriharmonic.

Now we are going to change slightly the notation for the one used in $[\mathrm{BDH}]$ and [BBDHPT]. Take all vector fields

$$
X_{\alpha}^{1}, X_{\alpha}^{2}, \quad \text { for } \alpha \in \bar{Q}_{i j}
$$

and denote them

Corresponding vectors

$$
X_{i j}^{\beta}, \quad 1 \leq \beta \leq d .
$$

$$
Y_{\alpha}^{1}, Y_{\alpha}^{2}
$$


denote by

$$
Y_{i j}^{\beta}
$$

using the same ordering. Analogously we change

$$
\mathcal{X}_{\alpha}, \mathcal{Y}_{\alpha}
$$

for

$$
\mathcal{X}_{j}^{\beta}, \mathcal{Y}_{j}^{\beta}
$$

Hence we get the left-invariant vector fields:

$$
X_{j}, X_{i j}^{\beta}, \mathcal{X}_{j}^{\beta}, H_{j}, Y_{i j}^{\beta}, \mathcal{Y}_{j}^{\beta} .
$$

Finally, for the buildings blocks we adopt the notation

$$
\begin{aligned}
\mathcal{L}_{j}^{\beta} & =\Delta_{\mathcal{Z}_{\alpha}}, \\
\Delta_{i} & =\Delta_{Z_{i}}, \\
\Delta_{i j}^{\beta} & =\Delta_{Z_{\alpha}^{k}} .
\end{aligned}
$$

Then formulas (6.2) and (6.3) can be written as:

$$
\begin{aligned}
\mathcal{H}_{k} & =\mathcal{L}_{k}+2 \Delta_{k}+\sum_{\substack{1 \leq i<k \\
\beta}} \Delta_{i k}^{\beta}+\sum_{\substack{k<i \leq r \\
\beta}} \Delta_{k i}^{\beta}, \\
\Delta & =\sum_{i} \mathcal{L}_{i}+\sum_{i} \Delta_{i}+\sum_{\substack{1 \leq i<j \leq r \\
\beta}} \Delta_{i j}^{\beta},
\end{aligned}
$$

for $\mathcal{L}_{k}=\sum_{\beta} \mathcal{L}_{k}^{\beta}$. Furthermore by [DHMP], Theorem 1.23:

$$
\begin{aligned}
\Delta_{j} & =X_{j}^{2}+H_{j}^{2}-H_{j}, \\
\mathcal{L}_{j}^{\alpha} & =\left(\mathcal{X}_{j}^{\alpha}\right)^{2}+\left(\mathcal{Y}_{j}^{\alpha}\right)^{2}-H_{j}, \\
\Delta_{i j}^{\alpha} & =\left(X_{i j}^{\alpha}\right)^{2}+\left(Y_{i j}^{\alpha}\right)^{2}-H_{j} .
\end{aligned}
$$

From $[\mathrm{DHMP}]$ and $[\mathrm{BDH}]$ it follows that pluriharmonicity is equivalent to being annihilated by each building block $\mathcal{L}_{i}^{\beta}, \Delta_{i j}^{\beta}, \Delta_{i}$ separately, and that is just what we are going to prove about $f$ :

Theorem 6.6. Let $f$ be a real, bounded function on $S$, annihilated by $\Delta$ and by $\mathcal{H}_{j}$, for $j=1, \ldots, r$. Then

for all $i, j, \beta$.

$$
\Delta_{i} f=0, \quad \mathcal{L}_{j}^{\beta} f=0, \quad \Delta_{i j}^{\beta} f=0
$$

A simple calculation shows that as a linear combination of $\Delta$ and $\mathcal{H}_{j}$ 's we may obtain the Laplace-Beltrami operator $\Delta_{T}$ for the tube domain $T_{\Omega}=V+i \Omega$, which is identified with the subgroup $V S_{0}$ of the group $S$. Indeed,

$$
\Delta_{T}=\sum_{1 \leq j \leq r} \mathcal{H}_{j}-\Delta=\sum_{1 \leq j \leq r} \Delta_{j}+\sum_{\substack{1 \leq i<j \leq r \\ \beta}} \Delta_{i j}^{\beta}
$$


Given $\zeta \in \mathcal{Z}$, the function $f_{\zeta}(x y a)=f((\zeta, x) y a)$ becomes a function on the tube domain $T_{\Omega}$ and all the operators that are linear combinations of the blocks $\Delta_{i j}^{\beta}, \Delta_{i}$ have perfect sense as operators there. In particular using $\Delta, \Delta_{T}$ and Poisson integrals both on ${ }^{c} \mathcal{D}$ and on $T_{\Omega}$ we are able to prove that for fixed $\zeta, f_{\zeta}$ is the Poisson-Szegö integral on $T_{\Omega}$ and so using Theorem (1.2) we obtain

Theorem 6.7 ([BBDHPT], Theorem 3.1). Let $f$ be a bounded function on $S$ annihilated by $\Delta$ and by $\mathcal{H}_{j}$, for $j=1, \ldots, r$. Then

$$
\mathcal{H}_{j}^{T} f=0 \quad \text { for } j=1, \ldots, r
$$

and

$$
\mathcal{L}_{j} f=\sum_{\beta} \mathcal{L}_{j}^{\beta} f=0 \quad \text { for } j=1, \ldots, r
$$

where $\mathcal{H}_{j}^{T}=2 \Delta_{j}+\sum_{k<j} \sum_{\beta} \Delta_{k j}^{\beta}$ are strongly diagonal Hua operators for $V S_{0}$.

Proof of theorem (6.6). To prove the theorem we are going to use induction on the rank of cone. Assuming $r>1$, we show how to reduce the problem to the rank $r-1$.

First let us decompose the domain ${ }^{c} \mathcal{D}$. Take $\Omega^{r-1}$ to be the rank $r-1$ cone determined by the frame $c_{1}, \ldots, c_{r-1}$. The underlying space $V^{r-1}$ for $\Omega^{r-1}$ is the subspace

$$
V^{r-1}=\bigoplus_{1 \leq i \leq j<r} V_{i j}
$$

Next let us define

$$
\mathcal{Z}^{r-1}=\bigoplus_{j=1}^{r-1} \mathcal{Z}_{j}
$$

Then it is easily seen that $\mathcal{Z}^{r-1} \times \mathcal{Z}^{r-1}$ is mapped by $\Phi$ into $\left(V^{r-1}\right)^{\mathbb{C}}$. Moreover, $\Phi(\zeta, \zeta)$ belongs to $\Omega^{r-1}$ when $\zeta \in \mathcal{Z}^{r-1}$. So we may define the corresponding Siegel domain $\mathcal{D}^{r-1}$ as

$$
\mathcal{D}^{r-1}=\left\{(\zeta, z) \in \mathcal{Z}^{r-1} \times\left(V^{r-1}\right)^{\mathbb{C}}: \Im z-\Phi(\zeta, \zeta) \in \Omega^{r-1}\right\}
$$

Now let

$$
\mathfrak{h}_{r}=\mathcal{Z}_{r} \oplus\left(\bigoplus_{j<r} V_{j r}\right) \oplus\left(\bigoplus_{j<r} \mathfrak{n}_{j r}\right) \oplus V_{r r} .
$$

Looking at the corresponding brackets relations one can prove that $\mathfrak{h}_{r}$ is isomorphic to the Lie algebra of the Heisenberg group $\mathbb{H}^{m}$ with $m=\chi+(r-1) d$. Therefore

$$
\exp \mathfrak{h}_{r}=\mathbb{C}^{m} \times \mathbb{R}=\mathbb{H}^{m}
$$

( $V_{r r}$ is the center of the group).

Put $A_{r}=\exp \mathbb{R} H_{r}$ and $S_{r}=\exp \mathfrak{h}_{r} \cdot A_{r}$ then $S_{r}$ is a solvable group acting simple transitively on the classical Siegel half plane $\mathcal{D}_{r}$ which is biholomorphic equivalent to the complex ball in $\mathbb{C}^{m+1}$ (see also the next section). We can identify $S_{r}$ with $\mathcal{D}_{r}$. 
On the level of the Lie algebra we have the decomposition

$$
\mathfrak{s}=\mathfrak{s}^{r-1} \oplus \mathfrak{s}_{r},
$$

where

$$
\begin{aligned}
\mathfrak{s}^{r-1} & =\left(\bigoplus_{j<r} \mathcal{Z}_{j}\right) \oplus\left(\bigoplus_{1 \leq i \leq j<r} V_{i j}\right) \oplus\left(\underset{1 \leq i<j<r}{\bigoplus} \mathfrak{n}_{i j}\right) \oplus\left(\underset{1 \leq j<r}{\bigoplus} \mathfrak{a}_{0 j}\right), \\
\mathfrak{s}_{r} & =\mathcal{Z}_{r} \oplus\left(\bigoplus_{1 \leq j<r} V_{j r}\right) \oplus\left(\underset{1 \leq j<r}{\bigoplus} \mathfrak{n}_{j r}\right) \oplus V_{r r} \oplus \mathfrak{a}_{0 r} .
\end{aligned}
$$

$\mathfrak{s}^{r-1}$ is a subalgebra of $\mathfrak{s}$ and $\mathfrak{s}_{r}$ is an ideal. Therefore we can decompose the group onto semidirect product of a subgroup $S^{r-1}$ and normal subgroup $S_{r}$

$$
S=S^{r-1} S_{r}
$$

$S^{r-1}$ acts simply transitively on the Siegel domain $\mathcal{D}^{r-1}$ of the rank $r-1$.

The complex structure on $\mathfrak{s}_{r}$ (transferred from $\left.\mathcal{D}_{r}\right)$ is the restriction of the one on $\mathfrak{s}$. Hence:

$$
\begin{aligned}
\mathcal{J}\left(X_{r}\right) & =H_{r}, \\
\mathcal{J}\left(H_{r}\right) & =-X_{r}, \\
\mathcal{J}\left(X_{j r}^{\alpha}\right) & =Y_{j r}^{\alpha}, \\
\mathcal{J}\left(Y_{j r}^{\alpha}\right) & =-X_{j r}^{\alpha}, \\
\mathcal{J}\left(\mathcal{X}_{r}^{\alpha}\right) & =\mathcal{Y}_{r}^{\alpha}, \\
\mathcal{J}\left(\mathcal{Y}_{r}^{\alpha}\right) & =-\mathcal{X}_{r}^{\alpha} .
\end{aligned}
$$

Furthermore the basis

$$
\mathcal{X}_{r}^{\alpha}, \mathcal{Y}_{r}^{\alpha}, X_{j r}^{\alpha}, Y_{j r}^{\alpha}, X_{r}, H_{r}
$$

of $\mathfrak{s}_{r}$ is orthonormal with respect to the Riemannian form $g_{r}$ on $S_{r}$. We can compute Riemannian connection $\widetilde{\nabla}$ on $\mathfrak{s}_{r}$ :

Lemma 6.14. The Riemannian connection $\widetilde{\nabla}$ is given by

$$
\begin{aligned}
\widetilde{\nabla}_{H_{r}} H_{r} & =0, \\
\widetilde{\nabla}_{X_{j r}^{\alpha}} X_{j r}^{\alpha} & =\frac{1}{2} H_{r}, \\
\widetilde{\nabla}_{X_{r}} X_{r} & =H_{r}, \\
\widetilde{\nabla}_{Y_{i r}^{\alpha}} Y_{i r}^{\alpha} & =\frac{1}{2} H_{r}, \\
\widetilde{\nabla}_{\mathcal{X}_{r}^{\alpha}} \mathcal{X}_{r}^{\alpha} & =\frac{1}{2} H_{r}, \\
\widetilde{\nabla}_{\mathcal{Y}_{r}^{\alpha}} \mathcal{Y}_{r}^{\alpha} & =\frac{1}{2} H_{r} .
\end{aligned}
$$


Defining operators $\widetilde{\Delta}_{r}, \widetilde{\mathcal{L}}_{r}^{\alpha}$ and $\widetilde{\Delta}_{j r}^{\alpha}$ as in (3.31) and (6.5), with $\widetilde{\nabla}$ in place of $\nabla$, using formula (3.31) and lemma (6.14), we get:

$$
\begin{aligned}
\widetilde{\Delta}_{r} & =X_{r}^{2}+H_{r}^{2}-H_{r}, \\
\widetilde{\mathcal{L}}_{r}^{\alpha} & =\left(\mathcal{X}_{r}^{\alpha}\right)^{2}+\left(\mathcal{Y}_{r}^{\alpha}\right)^{2}-H_{r}, \\
\widetilde{\Delta}_{i r}^{\alpha} & =\left(X_{i r}^{\alpha}\right)^{2}+\left(Y_{i r}^{\alpha}\right)^{2}-H_{r} .
\end{aligned}
$$

Since the above operators act from the right, they have perfect sense both on $S_{r}$ and $S$, by (6.5) we may write:

$$
\begin{aligned}
\widetilde{\Delta}_{r}\left(f_{s^{\prime}}\right)\left(s_{r}\right) & =\left(\Delta_{r} f\right)\left(s^{\prime} s_{r}\right), \\
\widetilde{\mathcal{L}}_{r}^{\alpha}\left(f_{s^{\prime}}\right)\left(s_{r}\right) & =\left(\mathcal{L}_{r}^{\alpha} f\right)\left(s^{\prime} s_{r}\right), \\
\widetilde{\Delta}_{j r}\left(f_{s^{\prime}}\right)\left(s_{r}\right) & =\left(\Delta_{j r} f\right)\left(s^{\prime} s_{r}\right) .
\end{aligned}
$$

where $s^{\prime} \in S^{r-1}, s_{r} \in S_{r}$ and $f_{s^{\prime}}\left(s_{r}\right)=f\left(s^{\prime} s_{r}\right)$.

In the next section we are going to restrict operators $\mathcal{L}_{r}$ and $\mathcal{H}_{j}^{T}$ to left cosets of $S_{r}$ and to prove that left-hand sides of (6.16) are zero. But in view of (6.16) this means that $f$ is annihilated by the strongly diagonal Hua operators and the Laplace-Beltrami operator corresponding to the domain $\mathcal{D}^{r-1}$, which completes the proof of theorem (6.6).

\section{Bounded pluriharmonic functions on the Siegel upper half plane}

Now we are going to prove the last step of the main theorem. The idea of our proof is very similar to the proof of theorem 6.7 in $[\mathrm{BDH}]$. But we shall use somewhat different operators, and for this reason we have to strengthen some of the lemmas. For completeness we give the whole proof. We should mention, that notation used in this section is independent on the notation contained in the rest part of the paper.

We shall consider the classical Siegel domain $\mathcal{D}$ defined by

$$
\mathcal{D}=\left\{\left(z, z_{n+1}\right) \in \mathbb{C}^{n} \times \mathbb{C}: \Im z_{n+1}>\frac{1}{4}|z|^{2}\right\} .
$$

It is well-known, that $\mathcal{D}$ is biholomorphically equivalent to the unit ball in $\mathbb{C}^{n+1}$. Let $G$ denote the group of holomorphic isometries of $\mathcal{D}$. Take the Iwasawa decomposition of $G$ :

$$
G=\mathbb{H}^{n} A U\left(\mathbb{C}^{n}\right) .
$$

Then the elements $(\zeta, u) \in \mathbb{H}^{n}$ (Heisenberg group), $a \in A=\mathbb{R}^{+}$(dilatations group) and $\rho \in U\left(\mathbb{C}^{n}\right)$ (the group of unitary mappings of $\mathbb{C}^{n}$ ) act on $\mathcal{D}$ in the following way:

$$
\begin{aligned}
(\zeta, u) \cdot\left(z, z_{n+1}\right) & =\left(\zeta+z, u+z_{n+1}+\frac{1}{2} \Im\langle z, \zeta\rangle+i|\zeta|^{2}\right), \\
\delta_{a} \cdot\left(z, z_{n+1}\right) & =\left(\sqrt{a} z, a z_{n+1}\right), \\
\rho \cdot\left(z, z_{n+1}\right) & =\left(\rho(z), z_{n+1}\right) .
\end{aligned}
$$


The semidirect product of $\mathbb{H}^{n}$ and $A$ acts simply transitively on $\mathcal{D}$. Therefore we can identify the group $S=\mathbb{H}^{n} A$ with the domain $\mathcal{D}$ :

$$
S \ni s=(\zeta, u, a) \sim(\zeta, u, a) \cdot(0, i) \in \mathcal{D} .
$$

Let us denote by $\mathcal{X}_{j}, \mathcal{Y}_{j}, T$ left-invariant fields on $\mathbb{H}^{n}$, which in e (the identity element of $\left.\mathbb{H}^{n}\right)$ agree respectively with $\partial_{x_{j}}, \partial_{y_{j}}, \partial_{u}\left(\zeta_{j}=x_{j}+i y_{j}\right)$. Then the operators $\widetilde{\mathcal{X}}_{j}, \widetilde{\mathcal{Y}}, \widetilde{T}, H$ given by

$$
\begin{aligned}
\widetilde{\mathcal{X}}_{j} & =\sqrt{a} \mathcal{X}_{j}, \\
\widetilde{\mathcal{Y}}_{j} & =\sqrt{a} \mathcal{Y}_{j}, \\
\widetilde{T} & =a T, \\
H & =a \partial_{a},
\end{aligned}
$$

are left-invariant on the group $S$, and form a basis of the Lie algebra $\mathfrak{s}$ of $S$.

We are going to consider the following operators:

$$
\begin{aligned}
D & =\sum_{1 \leq j \leq k}\left(\left(\widetilde{\mathcal{X}}_{j}\right)^{2}+\left(\widetilde{\mathcal{Y}}_{j}\right)^{2}\right)-k H \\
L & =\sum_{k+1 \leq j \leq n}\left(\left(\widetilde{\mathcal{X}}_{j}\right)^{2}+\left(\widetilde{\mathcal{Y}}_{j}\right)^{2}\right)-(n-k) H+2\left(\widetilde{T}^{2}+H^{2}-H\right), \\
L_{B} & =\sum_{1 \leq j \leq n}\left(\left(\mathcal{X}_{j}\right)^{2}+\left(\mathcal{Y}_{j}\right)^{2}\right) .
\end{aligned}
$$

$D$ and $L$ are admissible operators (compare (6.5)), $L_{B}$ is called a sublaplacian on the Heisenberg group. All the operators are related by the following equation:

$$
D+L=a L_{B}+2 a^{2}\left(\partial_{u}^{2}+\partial_{a}^{2}\right)-a n \partial_{a} .
$$

Notice that if

$$
\left\{\widetilde{\mathcal{X}}_{j}, \widetilde{\mathcal{Y}}_{j}: j=1, \ldots, k\right\}=\left\{\mathcal{X}_{r}^{\alpha}, \mathcal{Y}_{r}^{\alpha}: \alpha=1, \ldots, \chi\right\}
$$

and

$$
\left\{\widetilde{\mathcal{X}}_{j}, \widetilde{\mathcal{Y}}_{j}: j=k+1, \ldots, n\right\}=\left\{X_{j r}^{\alpha}, Y_{j r}^{\alpha}: j=1, \ldots, r-1, \alpha=1, \ldots, d\right\},
$$

where $\mathcal{X}_{r}^{\alpha}, \mathcal{Y}_{r}^{\alpha}, X_{j r}^{\alpha}, Y_{j r}^{\alpha}$ are left invariant vector fields on $S$ and $S_{r}$ defined in section 6 , then $\mathcal{L}_{r}, \mathcal{H}_{r}^{T}$ restricted to $S_{r}$ are $D$ and $L$, respectively.

The goal of this section is to prove the following theorem:

Theorem 7.3. Let $F$ be a real bounded function on $\mathcal{D}$ annihilated by $D$ and $L$. Then $F$ is a pluriharmonic function, which implies that for every $j,\left(\widetilde{\mathcal{X}}_{j}^{2}+\widetilde{\mathcal{Y}}_{j}^{2}-H\right) F=0$ and $\left(\widetilde{T}^{2}+H^{2}-H\right) F=0$.

In terms of the previous section this theorem says that $F$ is annihilated by all building blocks: $\Delta_{j r}^{\alpha}$ and $\mathcal{L}_{r}^{\alpha}$. 
Being $D+L$ harmonic, $F$ can be written as Poisson integral of its boundary value $f \in L^{\infty}\left(\mathbb{H}^{n}\right)$

(see $[\mathrm{DH}],[\mathrm{R}])$.

$$
F((\zeta, u) a)=f *_{\mathbb{H}^{n}} P_{a}(\zeta, u)=: F_{a}(\zeta, u)
$$

Proposition 7.4. Let $F$ be any bounded function on $\mathcal{D}$ annihilated by $D$ and $L$, and let $f \in L^{\infty}\left(\mathbb{H}^{n}\right)$ be its boundary value. Then $f$ satisfies the following equation

$$
\left(L_{B}^{2}+n^{2} \partial_{u}^{2}\right) f(\zeta, u)=0 .
$$

Proof. This proposition is a straightforward consequence of the fact, that the family of functions $F_{a}$ tends in the distribution sense to the function $f$, when $a$ goes to 0 . The convergence is sufficient to "transfer" differential operators $L_{B}$ and $\partial_{u}$ to $f$. However our assumptions give us more, namely limits

$$
\lim _{a \rightarrow 0} \partial_{a}^{m} F_{a}
$$

exist in the distribution sense as well, for all nonnegative integer numbers $m$. To prove this put

$$
\bar{D}=\sum_{1 \leq j \leq k}\left(\left(\mathcal{X}_{j}\right)^{2}+\left(\mathcal{Y}_{j}\right)^{2}\right)
$$

Then using (7.1) and an observation that $\partial_{a}$ and $\bar{D}$ commute we have

which implies

$$
\partial_{a}^{m} F_{a}(\zeta, u)=\frac{1}{k^{m}} \bar{D}^{m} F_{a}(\zeta, u)
$$

$$
\lim _{a \rightarrow 0} \partial_{a}^{m} F_{a}(\zeta, u)=\lim _{a \rightarrow 0} \frac{1}{k^{m}} \bar{D}^{m} F_{a}(\zeta, u)=\frac{1}{k^{m}} \bar{D}^{m} f(\zeta, u) .
$$

Now we are able to deal with the operator $L$. Let

then by (7.1)

$$
\bar{L}=\sum_{k+1 \leq j \leq n}\left(\left(\mathcal{X}_{j}\right)^{2}+\left(\mathcal{Y}_{j}\right)^{2}\right)
$$

$$
0=\lim _{a \rightarrow 0}\left(\bar{L}+2 a\left(\partial_{u}^{2}+\partial_{a}^{2}\right)-(n-k) \partial_{a}\right) F_{a}(\zeta, u)=\left(\bar{L}-\frac{n-k}{k} \bar{D}\right) f(\zeta, u)
$$

Hence

$$
\bar{L} f(\zeta, u)=\frac{n-k}{k} \bar{D} f(\zeta, u)
$$

Notice that the operators $\bar{L}$ and $\bar{D}$ commute, so

$$
\bar{L}^{2} f(\zeta, u)=\bar{L} \frac{n-k}{k} \bar{D} f(\zeta, u)=\frac{n-k}{k} \overline{D L} f(\zeta, u)=\left(\frac{n-k}{k}\right)^{2} \bar{D}^{2} f(\zeta, u) .
$$

Therefore we easily check that

$$
L_{B}^{2} f(\zeta, u)=(\bar{L}+\bar{D})^{2} f(\zeta, u)=\frac{n^{2}}{k^{2}} \bar{D}^{2} f(\zeta, u) .
$$


Finally applying again (7.1), differentiating it with respect to $a$ and using formulas (7.6), (7.7) and (7.8) we conclude that

$$
\begin{aligned}
0 & =\lim _{a \rightarrow 0} \partial_{a}\left(\bar{L}+2 a\left(\partial_{u}^{2}+\partial_{a}^{2}\right)-(n-k) \partial_{a}\right) F_{a}(\zeta, u) \\
& =\lim _{a \rightarrow 0}\left(\partial_{a} \bar{L}+2\left(\partial_{u}^{2}+\partial_{a}^{2}\right)+2 a\left(\partial_{a} \partial_{u}^{2}+\partial_{a}^{3}\right)-(n-k) \partial_{a}^{2}\right) F_{a}(\zeta, u) \\
& =\left(\frac{1}{k} \overline{L D}+2 \partial_{u}^{2}+\frac{2}{k^{2}} \bar{D}^{2}-\frac{n-k}{k^{2}} \bar{D}^{2}\right) f(\zeta, u) \\
& =\left(2 \partial_{u}^{2}+\left(\frac{n-k}{k^{2}}+\frac{2}{k^{2}}-\frac{n-k}{k^{2}}\right) \bar{D}^{2}\right) f(\zeta, u) \\
& =\frac{2}{n^{2}}\left(n^{2} \partial_{u}^{2}+L_{B}^{2}\right) f(\zeta, u),
\end{aligned}
$$

which complete the proof.

Now the strategy is to reduce the problem to functions $F$ whose boundary value are easier to handle, due to special properties of their Fourier transforms. First, we may clearly assume that

$$
f=\rho * \tilde{f},
$$

for $\tilde{f} \in L^{\infty}\left(\mathbb{H}^{n}\right), \rho \in \mathcal{S}\left(\mathbb{H}^{n}\right)$. Indeed, $F_{a}$ may be approximated by functions $\rho_{n} * F_{a}$ and on the level of boundary values this means (7.9). Furthermore, we prove the following

Proposition 7.10. Let $F$ be as in theorem (7.3) and let $f=\rho * \widetilde{f}\left(\widetilde{f} \in L^{\infty}\left(\mathbb{H}^{n}\right)\right.$, $\rho \in \mathcal{S}\left(\mathbb{H}^{n}\right)$ ) be its boundary value. Denote by $f(\zeta, \widehat{\lambda})$ the distributional partial Fourier transform of $f$ along $\mathbb{R}$. Then there exists a sequence of functions $\eta_{n} \in C_{c}^{\infty}(\mathbb{R} \backslash\{0\})$ such that for $f_{n}$ and $F_{n}$ defined by:

$$
\begin{aligned}
f_{n}(\zeta, u) & =\eta_{n} *_{\mathbb{R}} f(\zeta, u), \\
F_{n}(\zeta, u, a) & =\left(f_{n}\right) *_{\mathbb{H}^{n}} P_{a}(\zeta, u)=\eta_{n} *_{\mathbb{R}} F(\zeta, u, a),
\end{aligned}
$$

the following conditions hold:

- $F_{n}$ is annihilated by $L$ and $D$;

- $\operatorname{supp}_{n}(\cdot, \cdot) \subset \mathbb{C}^{n} \times\left\{\lambda \in \mathbb{R}: \varepsilon_{n}<|\lambda|<M_{n}\right\}$ and the constants $\varepsilon_{n}$ and $M_{n}$ are dependent only on $\eta_{n}$;

- the sequence $F_{n}$ tends to $F+c$.

It is clear that for any sequence of functions $\eta_{n}$ such that $\widehat{\eta}_{n} \in C_{c}^{\infty}(\mathbb{R} \backslash\{0\})$ the first two conditions are true. The main difficulties is to build a sequence that ensures the last condition.

Let $\phi$ be a Schwartz function on $\mathbb{R}$ such that

$$
\widehat{\phi}(\lambda)=\left\{\begin{array}{lll}
1 & \text { for } \quad|\lambda| \leq 1 \\
0 & \text { for } \quad|\lambda| \geq 2
\end{array}\right.
$$

and $\widehat{\phi}(\lambda)=\widehat{\phi}(-\lambda)$. 
For a given sequence $\left\{k_{n}\right\}_{n=1,2, \ldots}$ of natural numbers tending to infinity, let

$$
\psi_{n}(u)=\frac{1}{k_{n}} \phi\left(\frac{u}{k_{n}}\right) .
$$

Given a bounded function $g$ on $\mathbb{R}$, there is $\left\{k_{n}\right\}_{n=1,2, \ldots}, k_{n} \rightarrow \infty$ such that

$$
\lim _{n \rightarrow+\infty}\left\langle g, \psi_{n}\right\rangle \text { exists. }
$$

But by $[\mathrm{BDH}]$ (lemma 4.4) even more is true. We can choose a sequence $\left\{k_{n}\right\}_{n=1,2, \ldots}$ such that

$$
\lim _{n \rightarrow+\infty}\left\langle f_{\zeta}, \psi_{n}\right\rangle \quad \text { exists for all } \zeta \in \mathbb{C}^{n} .
$$

Now using (7.5) we prove that the above limit does not depend on $\zeta$.

Lemma 7.13. The function

$$
\mathbb{C}^{n} \ni \zeta \mapsto H(\zeta)=\lim _{n \rightarrow+\infty}\left\langle f_{\zeta}, \psi_{n}\right\rangle
$$

is constant.

Proof. Consider the function $\widetilde{H}$ on $\mathbb{H}^{n}$ defined by

$$
\widetilde{H}(\zeta, u)=H(\zeta)
$$

Then $\widetilde{H} \in L^{\infty}\left(\mathbb{H}^{n}\right)$ and it is a boundary value of a smooth function $\widetilde{H}_{a} \in L^{\infty}(S)$, given by:

$$
\widetilde{H}_{a}(\zeta, u)=\widetilde{H} * \mathbb{H}^{n} P_{a}(\zeta, u)
$$

Observe that for every $u \in \mathbb{R}$,

$$
\lim _{n \rightarrow+\infty} \psi_{n} *_{\mathbb{R}} f_{\zeta}(u)=\lim _{n \rightarrow+\infty}\left\langle\psi_{n}, f_{\zeta}\right\rangle
$$

Indeed,

$$
\begin{aligned}
\lim _{n \rightarrow+\infty}\left|\left\langle\psi_{n}, f_{\zeta}\right\rangle-\psi_{n} *_{\mathbb{R}} f_{\zeta}(u)\right| & =\lim _{n \rightarrow+\infty}\left|\int \frac{1}{k_{n}}\left(\phi\left(\frac{y}{k_{n}}\right)-\phi\left(\frac{u-y}{k_{n}}\right)\right) f_{\zeta}(y) d y\right| \\
& \leq c \lim _{n \rightarrow+\infty} \int\left|\phi(y)-\phi\left(y-\frac{u}{k_{n}}\right)\right| d y \\
& =0 .
\end{aligned}
$$

Hence by the Lebesque dominated convergence theorem

$$
\begin{aligned}
\widetilde{H}_{a}(\zeta, u) & =\left(\lim _{n \rightarrow+\infty} \psi_{n} *_{\mathbb{R}} f .(\cdot)\right) *_{\mathbb{H}^{n}} P_{a}(\zeta, u)=\lim _{n \rightarrow+\infty}\left(\psi_{n} *_{\mathbb{R}} f *_{\mathbb{H}^{n}} P_{a}(\zeta, u)\right) \\
& =\lim _{n \rightarrow+\infty} \psi_{n} *_{\mathbb{R}} F_{a}(\zeta, u) .
\end{aligned}
$$

pointwise hence also in the distributional sense on $S$. Each of functions $\psi_{n} *_{\mathbb{R}} F_{a}(\zeta, u)$ is annihilated by $L$ and $D$, so $\widetilde{H}_{a}$ is as well. Therefore by proposition (7.4)

$$
\left(L_{B}^{2}+n^{2} \partial_{u}^{2}\right) \widetilde{H}(\zeta, u)=0
$$


but $\widetilde{H}$ is independent on $u$, thus

$$
\Delta_{\zeta}^{2} \widetilde{H}(\zeta, u)=\Delta_{\zeta}^{2} H(\zeta)=0
$$

where $\Delta_{\zeta}^{2}$ is the Laplacian on $\mathbb{C}^{n}=\mathbb{R}^{2 n}$.

Taking the Fourier transform of both sides we obtain

$$
|\lambda|^{4} \widehat{H}(\lambda)=0
$$

which means

$$
\operatorname{supp} \widehat{H} \subset\{0\} .
$$

Boundedness of $H$ forces it to be constant.

Proof of proposition (7.10). Let $\phi$ be a function defined by (7.11). We select the sequence $\left\{k_{n}\right\}$ such that the condition (7.12) was satisfied. Take $\eta_{n}$ to be

$$
\eta_{n}(x)=k_{n} \phi\left(k_{n} x\right)-\frac{1}{k_{n}} \phi\left(\frac{x}{k_{n}}\right) .
$$

It is easy to check that this is the required sequence of functions.

Thus we may assume that for a positive $\varepsilon, M$

$$
\operatorname{supp} f(\zeta, \widehat{\lambda}) \subset \mathbb{C}^{n} \times\{\lambda: \varepsilon<|\lambda|<M\}
$$

Let $\phi_{1}, \phi_{2} \in \mathcal{S}(\mathbb{R})$ be defined as follows:

$$
\begin{aligned}
& \widehat{\phi}_{1}(\lambda)= \begin{cases}1 & \text { for } \lambda \in\langle\varepsilon, M\rangle, \\
0 & \text { for } \lambda \notin\left\langle\frac{\varepsilon}{2}, 2 M\right\rangle,\end{cases} \\
& \widehat{\phi}_{2}(\lambda)=\widehat{\phi}_{1}(-\lambda)
\end{aligned}
$$

We may decompose functions $f$ and $F$ into the sum of two functions

$$
\begin{aligned}
f & =f_{1}+f_{2}, \\
F & =F_{1}+F_{2},
\end{aligned}
$$

where

$$
\begin{aligned}
f_{i}(\zeta, u) & =\phi_{i} *_{\mathbb{R}} f_{\zeta}(u), \\
F_{i}(\zeta, u, a) & =f_{i} *_{\mathbb{H}^{n}} P_{a}(\zeta, u),
\end{aligned}
$$

and we see that

$$
\begin{aligned}
& \left.\widehat{f}_{1}\right|_{\mathbb{R}^{+}}=\left.\widehat{f}\right|_{\mathbb{R}^{+}}, \\
& \left.\widehat{f}_{2}\right|_{\mathbb{R}^{-}}=\left.\widehat{f}\right|_{\mathbb{R}^{-}} .
\end{aligned}
$$

Furthermore

$$
\widehat{f}_{2}(-\lambda)=\overline{\hat{f}_{1}(\lambda)}
$$

which imply

$$
F_{2}=\bar{F}_{1} .
$$

So to prove theorem (7.3) it remains to show 
Theorem 7.14. $F_{1}$ is holomorphic.

To prove this theorem we shall need some elementary theory of unitary representations of the Heisenberg group for which we refer to $[\mathrm{T}]$.

Let $U_{\lambda}$ be the Schrödinger representation of $\mathbb{H}^{n}$, ([T], 1.2.1). In the underlying Hilbert space $\mathcal{H}_{\lambda}=L^{2}\left(\mathbb{R}^{n}\right)$ we consider the basis consisting of properly scaled Hermite functions $\xi_{\alpha}^{\lambda}(1.4 .18$ and section 2.1 of $[\mathrm{T}])$. Let

$$
\phi_{\alpha, \beta}^{\lambda}(\zeta, u)=\left(U_{(\zeta, u)}^{\lambda} \xi_{\alpha}^{\lambda}, \xi_{\beta}^{\lambda}\right)
$$

Then

$$
\phi_{\alpha, \beta}^{\lambda}(\zeta, u)=(2 \pi)^{n / 2} e^{i \lambda u} \Phi_{\alpha, \beta}(\sqrt{|\lambda|} \zeta),
$$

where $\Phi_{\alpha, \beta}$ are the special Hermite functions, ([T],1.4.19). $\Phi_{\alpha, \beta}$ functions belong to the Schwartz class on $\mathbb{C}^{n}$ and are eigenfunctions of the sublaplacian $L_{B}$ :

$$
L_{B} \phi_{\alpha, \beta}^{\lambda}(\zeta, u)=-(2|\alpha|+n)|\lambda| \phi_{\alpha, \beta}^{\lambda}(\zeta, u) .
$$

Let

$$
e_{k}^{\lambda}(\zeta, u)=\sum_{|\alpha|=k} \phi_{\alpha, \alpha}^{\lambda}(\zeta, u)
$$

and for $\phi \in C_{c}^{\infty}(\mathbb{R} \backslash\{0\})$

$$
\psi_{\phi}^{k}(\zeta, u)=\int_{\mathbb{R}} e_{k}^{\lambda}(\zeta, u) \phi(\lambda) d \lambda
$$

Then $\psi_{\phi}^{k} \in \mathcal{S}\left(\mathbb{C}^{n} \times \mathbb{R}\right)$ and by $(7.17)$

$$
L_{B} \psi_{\phi}^{k}=-(2 k+n) \psi_{\phi^{\prime}}^{k},
$$

where $\phi^{\prime}(\lambda)=|\lambda| \phi(\lambda)$.

We shall need more information about $\Phi_{\alpha, \alpha}$. Let $L_{k}$ be the $k$-th Laguerre polynomial, i.e.

$$
L_{k}(t) e^{-t}=\frac{1}{k !} \partial_{t}^{k}\left(e^{-t} t^{k}\right) .
$$

Given a multiindex $\alpha=\left(\alpha_{1}, \ldots, \alpha_{n}\right)$ and $\zeta \in \mathbb{C}^{n}$ let

$$
L_{\alpha}(\zeta)=L_{\alpha_{1}}\left(\frac{1}{2}\left|\zeta_{1}\right|^{2}\right) \cdot \ldots \cdot L_{\alpha_{n}}\left(\frac{1}{2}\left|\zeta_{n}\right|^{2}\right)
$$

Then

$$
\Phi_{\alpha, \alpha}(\zeta)=(2 \pi)^{-n / 2} L_{\alpha}(\zeta) e^{-\frac{1}{4}|\zeta|^{2}}
$$

(see [T] 1.4.20).

Lemma 7.23. For every $k \neq 0$ and $\phi \in C_{c}^{\infty}(\mathbb{R} \backslash\{0\})$

$$
\int_{\mathbb{H}^{n}} f_{1}(\eta, t) \overline{\psi_{\phi}^{k}(\eta, t)} d \eta d t=0
$$


Proof. Let $\phi \in C_{c}^{\infty}(\mathbb{R} \backslash\{0\})$ and $\widetilde{\phi}(\lambda)=\lambda^{-2} \phi(\lambda)$. Applying (7.20) and proposition (7.4) we get

$$
\begin{aligned}
0 & =\int_{\mathbb{H}^{n}}\left(L_{B}^{2}+n^{2} \partial_{t}^{2}\right) f_{1}(\eta, t) \overline{\psi_{\widetilde{\phi}}^{k}(\eta, t)} d \eta d t \\
& =\int_{\mathbb{H}^{n}} f_{1}(\eta, t) \overline{\left(L_{B}^{2}+n^{2} \partial_{t}^{2}\right) \psi_{\widetilde{\phi}}^{k}(\eta, t)} d \eta d t \\
& =\left((2 k+n)^{2}-n^{2}\right) \int_{\mathbb{H}^{n}} f_{1}(\eta, t) \overline{\psi_{\phi}^{k}(\eta, t)} d \eta d t \\
& =4 k(k+n) \int_{\mathbb{H}^{n}} f_{1}(\eta, t) \overline{\psi_{\phi}^{k}(\eta, t)} d \eta d t
\end{aligned}
$$

and the lemma follows.

Furthermore, $f_{1}$ translated by any element $(\zeta, u) \in \mathbb{H}^{n}$ on the left is the boundary value of $F_{1}$ translated on the left by $(\zeta, u)$. Therefore, repeating the above argument we obtain:

$$
f_{1} *_{\mathbb{H}^{n}} \psi_{\phi}^{k}(\zeta, u)=\int_{\mathbb{H}^{n}} f_{1}\left((\zeta, u)(\eta, t) \overline{\psi \bar{\psi}^{k}(\eta, t)} d \eta d t=0,\right.
$$

for $k \neq 0$ and $\phi \in C_{c}^{\infty}(\mathbb{R} \backslash\{0\})$.

Let us define

$$
g(\zeta, u)=\phi_{1} *_{\mathbb{R}} P_{a}(\zeta, u) .
$$

$e_{k}^{\lambda}$ are joint eigenfunctions of $L_{B}$ and $T$, and we can expand $g$ in terms of its spectral projections:

$$
g(\zeta, u)=(2 \pi)^{-n-1} \sum_{k=0}^{\infty} \int_{-\infty}^{\infty} g *_{\mathbb{H}^{n}} e_{k}^{\lambda}(\zeta, u)|\lambda|^{n} d \lambda
$$

(see theorem 2.1.1 [T]), where the above series converges in $L^{2}\left(\mathbb{H}^{n}\right)$ norm. We are going to prove that:

Proposition 7.27. The series

$$
\sum_{k=0}^{\infty} \int_{-\infty}^{\infty} g *_{\mathbb{H}^{n}} e_{k}^{\lambda}(\zeta, u)|\lambda|^{n} d \lambda
$$

converges in $L^{1}\left(\mathbb{H}^{n}\right)$.

Proof. To prove this proposition we will use the following property of the Laguerre functions $([\mathrm{BDH}])$ :

For every $l, p \in \mathbb{N}$ there exist $c=c(l, p)$ and $M=M(l, p)$ such that

$$
\int_{0}^{\infty}(1+t)^{l}\left|\partial_{t}^{p} L_{k}(t)\right|^{2} e^{-t} d t \leq c k^{M}
$$


To estimate $L^{1}\left(\mathbb{H}^{n}\right)$ norm of

$$
\int_{-\infty}^{\infty} g *_{\mathbb{H}^{n}} e_{k}^{\lambda}(\zeta, u)|\lambda|^{n} d \lambda
$$

let us notice first that

$$
g *_{\mathbb{H}^{n}} e_{k}^{\lambda}(\zeta, u)=\phi_{1} *_{\mathbb{R}} P_{a} *_{\mathbb{H}^{n}} e_{k}^{\lambda}(\zeta, u)=P_{a} *_{\mathbb{H}^{n}}\left(\phi_{1} *_{\mathbb{R}} e_{k}^{\lambda}\right)(\zeta, u)=\widehat{\phi}_{1}(\lambda) P_{a} *_{\mathbb{H}^{n}} e_{\lambda}^{k}(\zeta, u) .
$$

By (7.17) we get

$$
\begin{aligned}
\| \int_{-\infty}^{\infty} g * \mathbb{H}^{n} & e_{k}^{\lambda}(\zeta, u)|\lambda|^{n} d \lambda\left\|_{L^{1}\left(\mathbb{H}^{n}\right)}=\right\| \int_{-\infty}^{\infty}\left(P_{a} * \mathbb{H}^{n}\right. \\
= & \frac{1}{(2 k+n)^{N}}\left\|\int_{-\infty}^{\lambda}(\zeta, u) \widehat{\phi}_{1}(\lambda)|\lambda|^{n} d \lambda\right\|_{L^{1}\left(\mathbb{H}^{n}\right)} \\
= & \frac{1}{(2 k+n)^{N}}\left\|\int_{-\infty}^{\infty}\left(L_{B}^{N} P_{a} *_{\mathbb{H}^{n}} e_{k}^{\lambda}\right)(\zeta, u) \widehat{\phi}_{1}(\lambda)|\lambda|^{n-N} d \lambda\right\|_{L^{1}\left(\mathbb{H}^{n}\right)} \\
\leq & \frac{c}{(2 k+n)^{N}}\left\|P_{a}\right\|_{L^{1}\left(\mathbb{H}^{n}\right)}\left\|\int_{-\infty}^{\infty} \widehat{\phi}_{1}(\lambda) \widehat{\phi}_{k}^{\lambda}(\zeta, u)|\lambda|^{n-N} d \lambda\right\|_{L^{1}\left(\mathbb{H}^{n}\right)}
\end{aligned}
$$

For the last inequality we estimated $\left|L_{B}^{N} P_{a}\right|$ by $P_{a}$ pointwise due to Harnack's principle. To obtain the thesis it is enough to show that

$$
\left\|\psi_{\eta_{N}}^{k}\right\|_{L^{1}\left(\mathbb{H}^{n}\right)} \leq c_{N} k^{m}
$$

for some $m$, independent on $N$, where

$$
\eta_{N}(\lambda)=\widehat{\phi}_{1}(\lambda)|\lambda|^{n-N}
$$

According to (7.16) and (7.18) we can write

$$
\psi_{\eta_{N}}^{k}(\zeta, u)=\sum_{|\alpha|=k}(2 \pi)^{\frac{n}{2}} \int_{\mathbb{R}} e^{i \lambda u} \eta_{N}(\lambda) \Phi_{\alpha, \alpha}(\sqrt{\lambda} \zeta) d \lambda .
$$

Hence the Schwartz inequality, (7.22) and (7.29) yield

$$
\begin{aligned}
& \int_{\mathbb{H}^{n}}\left|\psi_{\eta_{N}}^{k}(\zeta, u)\right| d u d \zeta \leq c \int_{\mathbb{H}^{n}}\left(1+u^{2}\right)\left|\psi_{\eta_{N}}^{k}(\zeta, u)\right|^{2} d u d \zeta \\
& \leq c \sum_{|\alpha|=k}\left(\int_{\mathbb{C}^{n}} \int_{\mathbb{R}}\left|\partial_{\lambda}\left(\eta_{N}(\lambda) \Phi_{\alpha, \alpha}(\sqrt{\lambda} \zeta)\right)\right|^{2} d \zeta d \lambda+\int_{\mathbb{C}^{n}} \int_{\mathbb{R}}\left|\eta_{N}(\lambda) \Phi_{\alpha, \alpha}(\sqrt{\lambda} \zeta)\right|^{2} d \zeta d \lambda\right) \\
& \quad \leq c_{N} \sum_{|\alpha|=k} \int_{\mathbb{C}^{n} \times[\varepsilon, M]}\left(\left|\partial_{\lambda}\left(\Phi_{\alpha, \alpha}(\sqrt{\lambda} \zeta)\right)\right|^{2}+\left|\Phi_{\alpha, \alpha}(\sqrt{\lambda} \zeta)\right|^{2}\right) d \zeta d \lambda \leq c_{N} k^{m} .
\end{aligned}
$$

Now taking $N$ large enough we obtain (7.28).

Lemma 7.30. For every $a \in \mathbb{R}^{+}$, the function $\mathbb{H}^{n} \ni(\zeta, u) \mapsto P_{a}(\zeta, u)$ is radial. 
Proof. For $\rho \in U\left(\mathbb{C}^{n}\right)$ define

$$
\widetilde{P}(\zeta, u)=P(\rho \zeta, u)
$$

and

$$
\widetilde{P}_{a}(\zeta, u)=a^{-1} \widetilde{P}\left(\delta_{a^{-1}}(\zeta), u\right)
$$

Then

$$
\widetilde{P}_{a}(\zeta, u)=P_{a}(\rho \zeta, u) .
$$

We claim that $\widetilde{P}_{a}$ is annihilated by the operator $L+D$. For this we apply the fact that $L_{B}$ and $\partial_{u}$ commute with the action of $U(n)$ on $\mathbb{H}^{n}$

$$
\begin{aligned}
\left(a L_{B}+a^{2} \partial_{u}^{2}\right) \widetilde{P}_{a}(\zeta, u) & =\left(a L_{B}+a^{2} \partial_{u}^{2}\right) P_{a}(\rho \zeta, u) \\
& =\left(a n \partial_{a}-2 a^{2} \partial_{a}^{2}\right) P_{a}(\rho \zeta, u)=\left(a n \partial_{a}-2 a^{2} \partial_{a}^{2}\right) \widetilde{P}_{a}(\zeta, u) .
\end{aligned}
$$

Moreover, $\widetilde{P}_{a}$ is an approximate identity. These two properties uniquely define the Poisson kernel ([DH], corollary 3.13). Hence

$$
P_{a}(\zeta, u)=\widetilde{P}_{a}(\zeta, u)
$$

Since $e_{k}^{\lambda}$ are radial as well, we get

Corollary 7.31. For every $\lambda \neq 0, k \in \mathbb{N}$ :

$$
e_{k}^{\lambda} * P_{a}(\zeta, u)=P_{a} * e_{k}^{\lambda}(\zeta, u) .
$$

Now we are ready to expand $F_{1}$. Using (7.26), proposition (7.27) and the above corollary we have:

$$
\begin{aligned}
F_{1}(\zeta, u, a) & =f_{1} *_{\mathbb{H}^{n}}\left(\phi_{1} *_{\mathbb{R}} P_{a}\right)(\zeta, u) \\
& =f_{1} *_{\mathbb{H}^{n}}\left((2 \pi)^{-n-1} \sum_{k=0}^{\infty} \int_{\mathbb{R}} \phi_{1} *_{\mathbb{R}} P_{a} *_{\mathbb{H}^{n}} e_{k}^{\lambda}|\lambda|^{n} d \lambda\right)(\zeta, u) \\
& =(2 \pi)^{-n-1} \sum_{k=0}^{\infty} \int_{\mathbb{R}} f_{1} *_{\mathbb{H}^{n}}\left(P_{a} *_{\mathbb{H}^{n}} \phi_{1} *_{\mathbb{R}} e_{k}^{\lambda}\right)(\zeta, u)|\lambda|^{n} d \lambda \\
& =(2 \pi)^{-n-1} \sum_{k=0}^{\infty} \int_{\mathbb{R}} f_{1} *_{\mathbb{H}^{n}} e_{k}^{\lambda} *_{\mathbb{H}^{n}} P_{a}(\zeta, u) \widehat{\phi}_{1}(\lambda)|\lambda|^{n} d \lambda \\
& =(2 \pi)^{-n-1} \sum_{k=0}^{\infty} f_{1} * \psi_{\eta}^{k} * P_{a}(\zeta, u),
\end{aligned}
$$

where

Hence by (7.24)

$$
\eta(\lambda)=\widehat{\phi}_{1}(\lambda)|\lambda|^{n}
$$

$$
F_{1}(\zeta, u, a)=(2 \pi)^{-n-1} f_{1} *_{\mathbb{H}^{n}} P_{a} *_{\mathbb{H}^{n}} \psi_{\eta}^{0}(\zeta, u) .
$$


On the other hand, by [DHMP] (lemma 2.37),

$$
U_{P_{a}}^{\lambda} \xi_{0}=e^{-\lambda a} \xi_{0} .
$$

Therefore,

$$
\begin{aligned}
P_{a} * \psi_{\eta}^{0}(\zeta, u) & =\int_{\mathbb{H}^{n}} P_{a}(\eta, t)\left(\int_{\mathbb{R}} e_{0}^{\lambda}\left((\eta, t)^{-1}(\zeta, u)\right) \widehat{\phi}_{1}(\lambda)|\lambda|^{n} d \lambda\right) d \eta d t \\
& =\int_{\mathbb{R}}\left(\int_{\mathbb{H}^{n}} P_{a}(\eta, t) \phi_{0,0}^{\lambda}\left((\eta, t)^{-1}(\zeta, u)\right) d \eta d t\right) \widehat{\phi}_{1}(\lambda)|\lambda|^{n} d \lambda \\
& =\int_{\mathbb{R}}\left(U_{(\zeta, u)}^{\lambda} \xi_{0}^{\lambda}, U_{P_{a}}^{\lambda} \xi_{0}^{\lambda}\right) \widehat{\phi}_{1}(\lambda)|\lambda|^{n} d \lambda \\
& =\int_{\mathbb{R}} e^{-\lambda a} \phi_{0,0}^{\lambda}(\zeta, u) \widehat{\phi}_{1}(\lambda)|\lambda|^{n} d \lambda .
\end{aligned}
$$

So it suffices to prove that the function

$$
G(\zeta, u, a)=e^{-\lambda a} \phi_{0,0}^{\lambda}(\zeta, u)
$$

is holomorphic. We have,

$$
G(\zeta, u, a)=(2 \pi)^{-n / 2} e^{-\lambda a} e^{i \lambda x} e^{-\frac{1}{4} \lambda|\zeta|^{2}}=(2 \pi)^{-n / 2} e^{i \lambda\left(x+i a+\frac{1}{4} i|\zeta|^{2}\right)}=e^{i \lambda z}
$$

which proves theorem (7.14).

\section{APPENDIX}

In $[\mathrm{DHP}]$ and $[\mathrm{BBDHPT}]$ the authors introduced a geometric definition of the Hua system. The definition was written in terms of the curvature tensor $R$ of the domain, which allows to generalize Hua operators to any Kählerian manifold. On Hermitian symmetric spaces both definitions are equivalent, which is an consequence of Theorem 4.2, page 180 in [H1]. Explicitly, this theorem says that for $X, Y \in \mathfrak{p}$, at the base point $o$,

$$
R(X, Y)=-\left.a d[X, Y]\right|_{\mathfrak{p}} .
$$

Therefore, one can apply the adjoint representation of $\mathfrak{k}$ on $\mathfrak{p}$ to the Hua system $\mathcal{H}$, to express it using the curvature tensor. Finally, if we use non-degeneracy of the adjoint representation and the invariance of the system under the group of biholomorphic transformations of the domain, we get the formula from previous papers.

\section{REFERENCES}

[BBDHPT] A. Bonami, D. Buraczewski, E. Damek, A. Hulanicki, R. Penney, and B. Trojan. Hua system and pluriharmonicity for symmetric irreducible Siegel domains of type II. to appear in Journal of Functional Analysis.

[BDH] D. Buraczewski, E. Damek, and A. Hulanicki. Bounded pluriharmonic functions on symmetric irreducible Siegel domains. to appear in Mathematische Zeitschrift.

[BV] N. Berline and M. Vergne. Equations de hua et noyau de poisson. Lecture Notes in Math., 880:1-51, 1981. Springer - Verlag. 
[DH $] \quad$ E. Damek and A. Hulanicki. Boundaries for left-invariant subelliptic operators on semidirect products of nilpotent and abelian groups. J. Reine Angew. Math., 411:1-38, 1990.

[DHMP] E. Damek, A. Hulanicki, D. Müller, and M. Peloso. Pluriharmonic $H^{2}$ functions on symmetric irreducible Siegel domains. Geom. and Funct. Anal., 10:1090-1117, 2000.

[DHP] E. Damek, A. Hulanicki, and R. Penney. Hua operators on bounded homogeneous domains in $\mathbb{C}^{n}$ and alternative reproducing kernels for holomorphic functions. Journal of Functional Analysis, 151 (1):77-120, 1997.

[FK] J. Faraut and A. Korányi. Analysis On Symmetric Cones. Clarendon Press, Oxford, 1994.

[HC] Harish-Chandra. Discrete series for semisimple Lie groups II. Acta Math., 116:1-111, 1966.

[H1] S. Helgason. Differential Geometry, Lie Groups, and Symmetric Spaces. Academic Press, New York, 1962.

[H2] S. Helgason. Groups and Geometric Analysis. Academic Press, Orlando, 1984.

[H3] S. Helgason. Geometric Analysis on Symmetric Spaces. American Mathematical Society, Providence, 1994.

[Hua] L. K. Hua. Harmonic Analysis of Functions of Several Complex Variables in the Classical Domains. Science Press, Peking (1958), Amer. Math. Soc. Transl., Math. Monograph 6, 1963.

[JK] K. Johnson and A. Korányi. The Hua operators on bounded symmetric domains of tube type. Annals of Math., 111 (2):589-608, 1980.

[J1] K. Johnson. Remarks on the theorem of Korányi and Malliavin on the Siegel upper half-plane of rank two. Proc. Amer. Math. Soc., 67:351-356, 1977.

[J2] K. Johnson. Differential equations and the Bergman- Shilov boundary on the Siegel upper half-plane. Arkiv for Matematik, 16:95-108, 1978.

$[\mathrm{KM}] \quad$ A. Korányi and P. Malliavin. Poisson formula and compound diffusion associated to an overdetermined elliptic system on the Siegel halfplane of rank two. Acta Math., 134:185-209, 1975.

[Kn] A. W. Knapp. Lie Groups, Beyond an Introduction. Birkhäuser, Boston - Basel - Berlin, 1996.

[K] A. Korányi. Analysis and Geometry on Complex Homogeneous Domains, chapter Function Spaces on Bounded Symmetric Domains, pages 183-281. Birkhäuser, Boston - Basel - Berlin, 2000 .

[KV] A. Korányi and S. Vági. Rational inner functions on bounded symmetric domains. Trans. A. M. S., 254:179-193, 1979.

[KW] A. Korányi and J. Wolf. Realization of Hermitian symmetric spaces as generalized half-planes. Annals of Math., 81 (2):265-288, 1965.

[L] M. Lassalle. Les équations de Hua d'un domaine borné symétrique de tube type. Invent. Math., 77:129-161, 1984.

[R] A. Raugi. Fonctions harmoniques sur les groupes localement compact á base dénombrable. Bull. Soc. Math. France, Memoiré, 54:5-118, 1977.

[S] I. Satake. Algebraic structures of symmetric domains. Iwanami-Shoten and Princeton Univ. Press, 1980.

[T] S. Thangavelu. Harmonic Analysis on the Heisenberg Group. Birkhäuser, Boston - Basel Berlin, 1998.

Instytut Matematyczny, Uniwersytet Wroclawski, Plac Grunwaldzki 2/4, 50-384 WroCLAW, POLAND

E-mail address: dbura@math.uni.wroc.pl 